\title{
Canards Existence in FitzHugh-Nagumo and Hodgkin-Huxley Neuronal Models
}

\author{
Jean-Marc Ginoux ${ }^{1}$ and Jaume Llibre ${ }^{2}$ \\ ${ }^{1}$ Laboratoire LSIS, CNRS, UMR 7296, Université de Toulon, BP 20132, 83957 La Garde Cedex, France \\ ${ }^{2}$ Departament de Matemàtiques, Universitat Autònoma de Barcelona, 08193 Bellaterra, Barcelona, Spain \\ Correspondence should be addressed to Jean-Marc Ginoux; ginoux@univ-tln.fr
}

Received 5 August 2015; Accepted 3 December 2015

Academic Editor: Matjaz Perc

Copyright ( 2015 J.-M. Ginoux and J. Llibre. This is an open access article distributed under the Creative Commons Attribution License, which permits unrestricted use, distribution, and reproduction in any medium, provided the original work is properly cited.

\begin{abstract}
In a previous paper we have proposed a new method for proving the existence of "canard solutions" for three- and four-dimensional singularly perturbed systems with only one fast variable which improves the methods used until now. The aim of this work is to extend this method to the case of four-dimensional singularly perturbed systems with two slow and two fast variables. This method enables stating a unique generic condition for the existence of "canard solutions" for such four-dimensional singularly perturbed systems which is based on the stability of folded singularities (pseudo singular points in this case) of the normalized slow dynamics deduced from a well-known property of linear algebra. This unique generic condition is identical to that provided in previous works. Application of this method to the famous coupled FitzHugh-Nagumo equations and to the Hodgkin-Huxley model enables showing the existence of "canard solutions" in such systems.
\end{abstract}

\section{Introduction}

In the beginning of the eighties, Benoît and Lobry [1], Benoît [2], and then Benoit [3] in his $\mathrm{PhD}$ - thesis studied canard solutions in $\mathbb{R}^{3}$. In the article entitled "Systèmes LentsRapides dans $\mathbb{R}^{3}$ et Leurs Canards," Benoît [2, p. 170] proved the existence of canards solution for three-dimensional singularly perturbed systems with two slow variables and one fast variable while using "nonstandard analysis" according to a theorem which stated that canard solutions exist in such systems provided that the pseudo singular point (this concept has been originally introduced by Argémi [4]; see Section 2.8) of the slow dynamics, that is, of the reduced vector field, is of saddle-type. Nearly twenty years later, Szmolyan and Wechselberger [5] extended "Geometric Singular Perturbation Theory (see Fenichel [6, 7], O'Malley [8], Jones [9], and Kaper [10])" to canards problems in $\mathbb{R}^{3}$ and provided a "standard version" of Benoît's theorem [2]. Very recently, Wechselberger [11] generalized this theorem for $n$-dimensional singularly perturbed systems with $k$ slow variables and $m$ fast (1). The methods used by Szmolyan and Wechselberger [5] and Wechselberger [11] require implementing a "desingularization procedure" which can be summarized as follows: first, they compute the normal form of such singularly perturbed systems which is expressed according to some coefficients ( $a$ and $b$ for dimension three and $\widetilde{a}, \widetilde{b}$, and $\widetilde{c}_{j}$ for dimension four) depending on the functions defining the original vector field and their partial derivatives with respect to the variables. Secondly, they project the "desingularized vector field" (originally called "normalized slow dynamics" by Benoît [2, p. 166]) of such a normal form on the tangent bundle of the critical manifold. Finally, they evaluate the Jacobian of the projection of this "desingularized vector field" at the folded singularity (originally called pseudo singular points by Argémi [4, p. 336]). This leads Szmolyan and Wechselberger [5, p. 427] and Wechselberger [11, p. 3298] to a "classification of folded singularities (pseudo singular points)." Thus, they showed that for three-dimensional singularly perturbed systems such folded singularity is of saddl-type if the following condition is satisfied, $a<0$ while for four-dimensional singularly perturbed systems such folded singularity is of saddle-type if $\widetilde{a}<0$. Then, Szmolyan and Wechselberger [5, p. 439] and Wechselberger $[11$, p. 3304] established their Theorem 4.1. which states that "In the folded saddle and in the folded node case singular 
canards perturb to maximal canard for sufficiently small $\varepsilon$." However, in their works neither Szmolyan and Wechselberger [5] nor Wechselberger [11] did not provide (to our knowledge) the expression of these constants $(a$ and $\widetilde{a})$ which are necessary to state the existence of canard solutions in such systems.

In a previous paper entitled "Canards Existence in Memristor's Circuits" (see Ginoux and Llibre [12]) we first provided the expression of these constants and then showed that they can be directly determined starting from the normalized slow dynamics and not from the projection of the "desingularized vector field" of the normal form. This method enabled stating a unique "generic" condition for the existence of "canard solutions" for such three- and four-dimensional singularly perturbed systems which is based on the stability of folded singularities of the normalized slow dynamics deduced from a well-known property of linear algebra. This unique condition which is completely identical to that provided by Benoît [2] and then by Szmolyan and Wechselberger [5] and finally by Wechselberger [11] is "generic" since it is exactly the same for singularly perturbed systems of dimensions three and four with only one fast variable.

The aim of this work is to extend this method to the case of four-dimensional singularly perturbed systems with $k=2$ slow and $m=2$ fast variables. Since the dimension of the system is $m=k+m$, such problem is known as "canards existence in $\mathbb{R}^{2+2}$." Moreover, in this particular case, where $k=m=2$, the folded singularities of Wechselberger [11, $\mathrm{p}$. 3298] are nothing else but the pseudo singular points of the late Argémi [4] as we will see below. Following the previous works, we show that for such four-dimensional singularly perturbed systems pseudo singular points are of saddle-type if $\widetilde{a}<0$. Then, according to Theorem 4.1. of Wechselberger [11, p. 3304] we provide the expression of this constant $\widetilde{a}$ which is necessary to establish the existence of canard solutions in such systems. So, we can state that the condition $\tilde{a}<0$ for existence of canards in such $\mathbb{R}^{2+2}$ is "generic" since it is exactly the same for singularly perturbed systems of dimensions three and four with only one fast variable.

The outline of this paper is as follows. In Section 1, definitions of singularly perturbed system, critical manifold, reduced system, "constrained system," canard cycles, folded singularities, and pseudo singular points are recalled. The method proposed in this paper is presented in Section 2 for the case of four-dimensional singularly perturbed systems with two fast variables. In Section 3, applications of this method to the famous coupled FitzHugh-Nagumo equations and to the Hodgkin-Huxley model enable showing the existence of "canard solutions" in such systems.

\section{Definitions}

2.1. Singularly Perturbed Systems. According to Tikhonov [13], Jones [9], and Kaper [10] singularly perturbed systems are defined as

$$
\begin{aligned}
& \vec{x}^{\prime}=\varepsilon \vec{f}(\vec{x}, \vec{y}, \varepsilon), \\
& \vec{y}^{\prime}=\vec{g}(\vec{x}, \vec{y}, \varepsilon),
\end{aligned}
$$

where $\vec{x} \in \mathbb{R}^{k}, \vec{y} \in \mathbb{R}^{m}, \varepsilon \in \mathbb{R}^{+}$, and the prime denotes differentiation with respect to the independent variable $t^{\prime}$. The functions $\vec{f}$ and $\vec{g}$ are assumed to be $C^{\infty}$ functions (in certain applications these functions will be supposed to be $C^{r}$, $r \geqslant 1$ ) of $\vec{x}, \vec{y}$, and $\varepsilon$ in $U \times I$, where $U$ is an open subset of $\mathbb{R}^{k} \times \mathbb{R}^{m}$ and $I$ is an open interval containing $\varepsilon=0$.

In the case when $0<\varepsilon \ll 1$, that is, $\varepsilon$ is a small positive number, the variable $\vec{x}$ is called slow variable and $\vec{y}$ is called fast variable. Using Landau's notation, $O\left(\varepsilon^{p}\right)$ represents a function $f$ of $u$ and $\varepsilon$ such that $f(u, \varepsilon) / \varepsilon^{p}$ is bounded for positive $\varepsilon$ going to zero, uniformly for $u$ in the given domain.

In general we consider that $\vec{x}$ evolves at an $O(\varepsilon)$ rate while $\vec{y}$ evolves at an $O(1)$ slow rate. Reformulating system (1) in terms of the rescaled variable $t=\varepsilon t^{\prime}$, we obtain

$$
\begin{aligned}
\dot{\vec{x}} & =\vec{f}(\vec{x}, \vec{y}, \varepsilon), \\
\varepsilon \dot{\vec{y}} & =\vec{g}(\vec{x}, \vec{y}, \varepsilon) .
\end{aligned}
$$

The dot represents the derivative with respect to the new independent variable $t$.

The independent variables $t^{\prime}$ and $t$ are referred to the fast and slow times, respectively, and (1) and (2) are called the fast and slow systems, respectively. These systems are equivalent whenever $\varepsilon \neq 0$, and they are labeled singular perturbation problems when $0<\varepsilon \ll 1$. The label "singular" stems in part from the discontinuous limiting behavior in system (1) as $\varepsilon \rightarrow 0$.

2.2. Reduced Slow System. In such case system (2) leads to a differential-algebraic system (DAE) called reduced slow system whose dimension decreases from $k+m=n$ to $m$. Then, the slow variable $\vec{x} \in \mathbb{R}^{k}$ partially evolves in the submanifold $M_{0}$ called the critical manifold (it represents the approximation of the slow invariant manifold, with an error of $O(\varepsilon)$ ). The reduced slow system is

$$
\begin{aligned}
\dot{\vec{x}} & =\vec{f}(\vec{x}, \vec{y}, \varepsilon), \\
\overrightarrow{0} & =\vec{g}(\vec{x}, \vec{y}, \varepsilon) .
\end{aligned}
$$

2.3. Slow Invariant Manifold. The critical manifold is defined by

$$
M_{0}:=\{(\vec{x}, \vec{y}): \vec{g}(\vec{x}, \vec{y}, 0)=\overrightarrow{0}\}
$$

Such a normally hyperbolic invariant manifold (4) of the reduced slow system (3) persists as a locally invariant slow manifold of the full problem (1) for $\varepsilon$ sufficiently small. This locally slow invariant manifold is $O(\varepsilon)$ close to the critical manifold.

When $D_{\vec{x}} \vec{f}$ is invertible, thanks to the Implicit Function Theorem, $M_{0}$ is given by the graph of a $C^{\infty}$ function $\vec{x}=$ $\vec{G}_{0}(\vec{y})$ for $\vec{y} \in D$, where $D \subseteq \mathbb{R}^{k}$ is a compact, simply connected domain and the boundary of $D$ is a $(k-1)$ dimensional $C^{\infty}$ submanifold (the set $D$ is overflowing invariant with respect to (2) when $\varepsilon=0$; see Kaper [10] and Jones [9]). 
According to Fenichel $[6,7]$ theory if $0<\varepsilon \ll 1$ is sufficiently small, then there exists a function $\vec{G}(\vec{y}, \varepsilon)$ defined on $D$ such that the manifold,

$$
M_{\varepsilon}:=\{(\vec{x}, \vec{y}): \vec{x}=\vec{G}(\vec{y}, \varepsilon)\},
$$

is locally invariant under the flow of system (1). Moreover, there exist perturbed local stable (or attracting) $M_{a}$ and unstable (or repelling) $M_{r}$ branches of the slow invariant manifold $M_{\varepsilon}$. Thus, normal hyperbolicity of $M_{\varepsilon}$ is lost via a saddle-node bifurcation of the reduced slow system (3). Then, it gives rise to solutions of "canard" type.

2.4. Canards: Singular Canards and Maximal Canards. A canard is a solution of singularly perturbed dynamical system (1) following the attracting branch $M_{a}$ of the slow invariant manifold, passing near a bifurcation point located on the fold of this slow invariant manifold, and then following the repelling branch $M_{r}$ of the slow invariant manifold.

A singular canard is a solution of reduced slow system (3) following the attracting branch $M_{a, 0}$ of the critical manifold, passing near a bifurcation point located on the fold of this critical manifold, and then following the repelling branch $M_{r, 0}$ of the critical manifold.

A maximal canard corresponds to the intersection of the attracting and repelling branches $M_{a, \varepsilon} \cap M_{r, \varepsilon}$ of the slow manifold in the vicinity of a nonhyperbolic point.

According to Wechselberger [11, p. 3302],

"Such a maximal canard defines a family of canards nearby which are exponentially close to the maximal canard, i.e., a family of solutions of (1) that follow an attracting branch $M_{a, \varepsilon}$ of the slow manifold and then follow, rather surprisingly, a repelling/saddle branch $M_{r, \varepsilon}$ of the slow manifold for a considerable amount of slow time. The existence of this family of canards is a consequence of the non-uniqueness of $M_{a, \varepsilon}$ and $M_{r, \varepsilon}$. However, in the singular limit $\varepsilon \rightarrow 0$, such a family of canards is represented by a unique singular canard."

Canards are special class of solutions of singularly perturbed dynamical systems for which normal hyperbolicity is lost. Canards in singularly perturbed systems with two or more slow variables $\left(\vec{x} \in \mathbb{R}^{k}, k \geqslant 2\right)$ and one fast variable $\left(\vec{y} \in \mathbb{R}^{m}, m=1\right)$ are robust, since maximal canards generically persist under small parameter changes (see Benoît $[2,14]$, Szmolyan and Wechselberger [5], and Wechselberger $[11,15])$.

2.5. Constrained System. In order to characterize the "slow dynamics," that is, the slow trajectory of the reduced slow system (3) (obtained by setting $\varepsilon=0$ in (2)), Takens [16] introduced the "constrained system" defined as follows:

$$
\begin{aligned}
\dot{\vec{x}} & =\vec{f}(\vec{x}, \vec{y}, 0), \\
D_{\vec{y}} \vec{g} \cdot \dot{\vec{y}} & =-\left(D_{\vec{x}} \vec{g} \cdot \vec{f}\right)(\vec{x}, \vec{y}, 0), \\
\overrightarrow{0} & =\vec{g}(\vec{x}, \vec{y}, 0) .
\end{aligned}
$$

Since, according to Fenichel $[6,7]$, the critical manifold $\vec{g}(\vec{x}, \vec{y}, 0)$ may be considered as locally invariant under the flow of system (1), we have

$$
\frac{d \vec{g}}{d t}(\vec{x}, \vec{y}, 0)=0 \Longleftrightarrow D_{\vec{x}} \vec{g} \cdot \dot{\vec{x}}+D_{\vec{y}} \vec{g} \cdot \dot{\vec{y}}=\overrightarrow{0} .
$$

By replacing $\dot{\vec{x}}$ by $\vec{f}(\vec{x}, \vec{y}, 0)$ leads to

$$
D_{\vec{x}} \vec{g} \cdot \vec{f}(\vec{x}, \vec{y}, 0)+D_{\vec{y}} \vec{g} \cdot \dot{\vec{y}}=\overrightarrow{0} .
$$

This justifies the introduction of the constrained system.

Now, let $\operatorname{adj}\left(D_{\vec{y}} \vec{g}\right)$ denote the adjoint of the matrix $D_{\vec{y}} \vec{g}$ which is the transpose of the cofactor matrix $D_{\vec{y}} \vec{g}$; then while multiplying the left-hand side of (6) by the inverse matrix $\left(D_{\vec{y}} \vec{g}\right)^{-1}$ obtained by the adjoint method we have

$$
\begin{aligned}
\dot{\vec{x}} & =\vec{f}(\vec{x}, \vec{y}, 0), \\
\operatorname{det}\left(D_{\vec{y}} \vec{g}\right) \dot{\vec{y}} & =-\left(\operatorname{adj}\left(D_{\vec{y}} \vec{g}\right) \cdot D_{\vec{x}} \vec{g} \cdot \vec{f}\right)(\vec{x}, \vec{y}, 0), \\
\overrightarrow{0} & =\vec{g}(\vec{x}, \vec{y}, 0) .
\end{aligned}
$$

2.6. Normalized Slow Dynamics. Then, by rescaling the time by setting $t=-\operatorname{det}\left(D_{\vec{y}} \vec{g}\right) \tau$ we obtain the following system which has been called by Benoît [2, p. 166] "normalized slow dynamics":

$$
\begin{aligned}
& \dot{\vec{x}}=-\operatorname{det}\left(D_{\vec{y}} \vec{g}\right) \vec{f}(\vec{x}, \vec{y}, 0), \\
& \dot{\vec{y}}=\left(\operatorname{adj}\left(D_{\vec{y}} \vec{g}\right) \cdot D_{\vec{x}} \vec{g} \cdot \vec{f}\right)(\vec{x}, \vec{y}, 0), \\
& \overrightarrow{0}=\vec{g}(\vec{x}, \vec{y}, 0),
\end{aligned}
$$

where the overdot now denotes the time derivation with respect to $\tau$.

Let us notice that Argémi [4] proposed to rescale time by setting $t=-\operatorname{det}\left(D_{\vec{y}} \vec{g}\right) \operatorname{sgn}\left(\operatorname{det}\left(D_{\vec{y}} \vec{g}\right)\right) \tau$ in order to keep the same flow direction in (10) as in (9).

2.7. Desingularized Vector Field. By application of the Implicit Function Theorem, let suppose that we can explicitly express from (4), say, without loss of generality, $x_{1}$ as a function $\phi_{1}$ of the other variables. This implies that $M_{0}$ is locally the graph of a function $\phi_{1}: \mathbb{R}^{k} \rightarrow \mathbb{R}^{m}$ over the base $U=(\vec{\chi}, \vec{y})$, where $\vec{\chi}=\left(x_{2}, x_{3}, \ldots, x_{k}\right)$. Thus, we can span the "normalized slow dynamics" on the tangent bundle at the critical manifold $M_{0}$ at the pseudo singular point. This leads to the so-called desingularized vector field:

$$
\begin{aligned}
& \dot{\vec{\chi}}=-\operatorname{det}\left(D_{\vec{y}} \vec{g}\right) \vec{f}(\vec{\chi}, \vec{y}, 0), \\
& \dot{\vec{y}}=\left(\operatorname{adj}\left(D_{\vec{y}} \vec{g}\right) \cdot D_{\vec{x}} \vec{g} \cdot \vec{f}\right)(\vec{\chi}, \vec{y}, 0) .
\end{aligned}
$$

2.8. Pseudo Singular Points and Folded Singularities. As recalled by Guckenheimer and Haiduc [17, p. 91], pseudo singular points have been introduced by the late Argémi [4] for low-dimensional singularly perturbed systems and are 
defined as singular points of the "normalized slow dynamics" (10). Twenty-three years later, Szmolyan and Wechselberger [5, p. 428] called such pseudo singular points folded singularities. In a recent publication entitled "A Propos de Canards" Wechselberger [11, p. 3295] proposed to define such singularities for $n$-dimensional singularly perturbed systems with $k$ slow variables and $m$ fast as the solutions of the following system:

$$
\begin{aligned}
\operatorname{det}\left(D_{\vec{y}} \vec{g}\right) & =0, \\
\left(\operatorname{adj}\left(D_{\vec{y}} \vec{g}\right) \cdot D_{\vec{x}} \vec{g} \cdot \vec{f}\right)(\vec{x}, \vec{y}, 0) & =\overrightarrow{0}, \\
\vec{g}(\vec{x}, \vec{y}, 0) & =\overrightarrow{0} .
\end{aligned}
$$

Thus, for dimensions higher than three, his concept encompasses that of Argémi. Moreover, Wechselberger [11, p. 3296] proved that folded singularities form a $(k-2)$ dimensional manifold. Thus, for $k=2$ the folded singularities are nothing else than the pseudo singular points defined by Argémi [4], while for $k \geqslant 3$ the folded singularities are no more points but a $(k-2)$-dimensional manifold. Moreover, let us notice on one hand that the original system (1) includes $n=k+m$ variables and on the other hand that system (12) comprises $p=2 m+1$ equations. However, in the particular case $k=m=2$, two equations of system (12) are linearly dependent. So, such system only comprises $p=2 m=2 k$ equations. So, all the variables (unknowns) of system (12) can be determined. The solutions of this system are called pseudo singular points. We will see in Section 3 that the stability analysis of these pseudo singular points will give rise to a condition for the existence of canard solutions in the original system (1).

\section{Four-Dimensional Singularly Perturbed Systems with Two Fast Variables}

Four-dimensional singularly perturbed dynamical system (2) with $k=2$ slow variables and $m=2$ fast may be written as

$$
\begin{aligned}
\dot{x}_{1} & =f_{1}\left(x_{1}, x_{2}, y_{1}, y_{2}\right), \\
\dot{x}_{2} & =f_{2}\left(x_{1}, x_{2}, y_{1}, y_{2}\right), \\
\varepsilon \dot{y}_{1} & =g_{1}\left(x_{1}, x_{2}, y_{1}, y_{2}\right), \\
\varepsilon \dot{y}_{2} & =g_{2}\left(x_{1}, x_{2}, y_{1}, y_{2}\right),
\end{aligned}
$$

where $\vec{x}=\left(x_{1}, x_{2}\right)^{t} \in \mathbb{R}^{2}, \vec{y}=\left(y_{1}, y_{2}\right) \in \mathbb{R}^{2}, 0<\varepsilon \ll 1$, and the functions $f_{i}$ and $g_{i}$ are assumed to be $C^{2}$ functions of $\left(x_{1}, x_{2}, y_{1}, y_{2}\right)$.

3.1. Critical Manifold. The critical manifold equation of system (13a), (13b), (13c), and (13d) is defined by setting $\varepsilon=0$ in $((13 \mathrm{c})$ and $(13 \mathrm{~d}))$. Thus, we obtain

$$
\begin{aligned}
& g_{1}\left(x_{1}, x_{2}, y_{1}, y_{2}\right)=0, \\
& g_{2}\left(x_{1}, x_{2}, y_{1}, y_{2}\right)=0 .
\end{aligned}
$$

By application of the Implicit Function Theorem, let us suppose that we can explicitly express from ((14a) and (14b)), say, without loss of generality, $x_{1}$ and $y_{1}$ as functions of the other variables:

$$
\begin{aligned}
& x_{1}=\phi_{1}\left(x_{2}, y_{1}, y_{2}\right), \\
& y_{1}=\phi_{2}\left(x_{1}, x_{2}, y_{2}\right) .
\end{aligned}
$$

3.2. Constrained System. The constrained system is obtained by equating with zero the time derivative of $g_{1,2}\left(x_{1}, x_{2}, y_{1}\right.$, $\left.y_{2}\right)$ :

$$
\begin{aligned}
& \frac{d g_{1}}{d t}=\frac{\partial g_{1}}{\partial x_{1}} \dot{x}_{1}+\frac{\partial g_{1}}{\partial x_{2}} \dot{x}_{2}+\frac{\partial g_{1}}{\partial y_{1}} \dot{y}_{1}+\frac{\partial g_{1}}{\partial y_{1}} \dot{y}_{2}=0 \\
& \frac{d g_{2}}{d t}=\frac{\partial g_{2}}{\partial x_{1}} \dot{x}_{1}+\frac{\partial g_{2}}{\partial x_{2}} \dot{x}_{2}+\frac{\partial g_{2}}{\partial y_{1}} \dot{y}_{1}+\frac{\partial g_{2}}{\partial y_{1}} \dot{y}_{2}=0 .
\end{aligned}
$$

Equations (16a) and (16b) may be written as

$$
\begin{aligned}
& \frac{\partial g_{1}}{\partial y_{1}} \dot{y}_{1}+\frac{\partial g_{1}}{\partial y_{1}} \dot{y}_{2}=-\left(\frac{\partial g_{1}}{\partial x_{1}} \dot{x}_{1}+\frac{\partial g_{1}}{\partial x_{2}} \dot{x}_{2}\right), \\
& \frac{\partial g_{2}}{\partial y_{1}} \dot{y}_{1}+\frac{\partial g_{2}}{\partial y_{1}} \dot{y}_{2}=-\left(\frac{\partial g_{2}}{\partial x_{1}} \dot{x}_{1}+\frac{\partial g_{2}}{\partial x_{2}} \dot{x}_{2}\right) .
\end{aligned}
$$

By solving the system of (17a) and (17b) with two unknowns $\left(\dot{y}_{1}, \dot{y}_{2}\right)$ we find

$$
\begin{aligned}
& \dot{y}_{1}=\frac{-\left(\left(\partial g_{1} / \partial x_{1}\right) \dot{x}_{1}+\left(\partial g_{1} / \partial x_{2}\right) \dot{x}_{2}\right)\left(\partial g_{2} / \partial y_{2}\right)+\left(\left(\partial g_{2} / \partial x_{1}\right) \dot{x}_{1}+\left(\partial g_{2} / \partial x_{2}\right) \dot{x}_{2}\right)\left(\partial g_{1} / \partial y_{2}\right)}{\operatorname{det}\left[J_{\left(y_{1}, y_{2}\right)}\right]}, \\
& \dot{y}_{2}=\frac{-\left(\left(\partial g_{1} / \partial x_{1}\right) \dot{x}_{1}+\left(\partial g_{1} / \partial x_{2}\right) \dot{x}_{2}\right)\left(\partial g_{2} / \partial y_{1}\right)+\left(\left(\partial g_{2} / \partial x_{1}\right) \dot{x}_{1}+\left(\partial g_{2} / \partial x_{2}\right) \dot{x}_{2}\right)\left(\partial g_{1} / \partial y_{1}\right)}{\operatorname{det}\left[J_{\left(y_{1}, y_{2}\right)}\right]} .
\end{aligned}
$$


So, we have the following constrained system:

$$
\begin{aligned}
\dot{x}_{1} & =f_{1}\left(x_{1}, x_{2}, y_{1}, y_{2}\right), \\
\dot{x}_{2} & =f_{2}\left(x_{1}, x_{2}, y_{1}, y_{2}\right), \\
\dot{y}_{1} & =\frac{-\left(\left(\partial g_{1} / \partial x_{1}\right) \dot{x}_{1}+\left(\partial g_{1} / \partial x_{2}\right) \dot{x}_{2}\right)\left(\partial g_{2} / \partial y_{2}\right)+\left(\left(\partial g_{2} / \partial x_{1}\right) \dot{x}_{1}+\left(\partial g_{2} / \partial x_{2}\right) \dot{x}_{2}\right)\left(\partial g_{1} / \partial y_{2}\right)}{\operatorname{det}\left[J_{\left(y_{1}, y_{2}\right)}\right]}, \\
\dot{y}_{2} & =\frac{-\left(\left(\partial g_{1} / \partial x_{1}\right) \dot{x}_{1}+\left(\partial g_{1} / \partial x_{2}\right) \dot{x}_{2}\right)\left(\partial g_{2} / \partial y_{1}\right)+\left(\left(\partial g_{2} / \partial x_{1}\right) \dot{x}_{1}+\left(\partial g_{2} / \partial x_{2}\right) \dot{x}_{2}\right)\left(\partial g_{1} / \partial y_{1}\right)}{\operatorname{det}\left[J_{\left(y_{1}, y_{2}\right)}\right]}, \\
0 & =g_{1}\left(x_{1}, x_{2}, y_{1}, y_{2}\right), \\
0 & =g_{2}\left(x_{1}, x_{2}, y_{1}, y_{2}\right) .
\end{aligned}
$$

3.3. Normalized Slow Dynamics. By rescaling the time by setting $t=-\operatorname{det}\left[J_{\left(y_{1}, y_{2}\right)}\right] \tau$ we obtain the "normalized slow dynamics":

$$
\begin{aligned}
\dot{x}_{1}= & -f_{1}\left(x_{1}, x_{2}, y_{1}, y_{2}\right) \operatorname{det}\left[J_{\left(y_{1}, y_{2}\right)}\right] \\
= & F_{1}\left(x_{1}, x_{2}, x_{3}, y_{1}\right), \\
\dot{x}_{2}= & -f_{2}\left(x_{1}, x_{2}, y_{1}, y_{2}\right) \operatorname{det}\left[J_{\left(y_{1}, y_{2}\right)}\right. \\
= & F_{2}\left(x_{1}, x_{2}, x_{3}, y_{1}\right), \\
\dot{y}_{1}= & \left(\frac{\partial g_{1}}{\partial x_{1}} \dot{x}_{1}+\frac{\partial g_{1}}{\partial x_{2}} \dot{x}_{2}\right) \frac{\partial g_{2}}{\partial y_{2}} \\
& -\left(\frac{\partial g_{2}}{\partial x_{1}} \dot{x}_{1}+\frac{\partial g_{2}}{\partial x_{2}} \dot{x}_{2}\right) \frac{\partial g_{1}}{\partial y_{2}}=G_{1}\left(x_{1}, x_{2}, x_{3}, y_{1}\right), \\
\dot{y}_{2}= & \left(\frac{\partial g_{1}}{\partial x_{1}} \dot{x}_{1}+\frac{\partial g_{1}}{\partial x_{2}} \dot{x}_{2}\right) \frac{\partial g_{2}}{\partial y_{1}} \\
& -\left(\frac{\partial g_{2}}{\partial x_{1}} \dot{x}_{1}+\frac{\partial g_{2}}{\partial x_{2}} \dot{x}_{2}\right) \frac{\partial g_{1}}{\partial y_{1}}=G_{2}\left(x_{1}, x_{2}, x_{3}, y_{1}\right), \\
0= & g_{1}\left(x_{1}, x_{2}, y_{1}, y_{2}\right), \\
0= & g_{2}\left(x_{1}, x_{2}, y_{1}, y_{2}\right),
\end{aligned}
$$

where the overdot now denotes the time derivation with respect to $\tau$.

3.4. Desingularized System on the Critical Manifold. Then, since we have supposed that $x_{1}$ and $y_{1}$ may be explicitly expressed as functions of the other variables ((15a) and (15b)), they can be used to project the normalized slow dynamics
(20) on the tangent bundle of the critical manifold. So, we have

$$
\begin{aligned}
\dot{x}_{2}= & -f_{2}\left(x_{1}, x_{2}, y_{1}, y_{2}\right) \operatorname{det}\left[J_{\left(y_{1}, y_{2}\right)}\right]=F_{2}\left(x_{2}, y_{2}\right), \\
\dot{y}_{2}= & \left(\frac{\partial g_{1}}{\partial x_{1}} \dot{x}_{1}+\frac{\partial g_{1}}{\partial x_{2}} \dot{x}_{2}\right) \frac{\partial g_{2}}{\partial y_{1}} \\
& -\left(\frac{\partial g_{2}}{\partial x_{1}} \dot{x}_{1}+\frac{\partial g_{2}}{\partial x_{2}} \dot{x}_{2}\right) \frac{\partial g_{1}}{\partial y_{1}}=G_{2}\left(x_{2}, y_{2}\right) .
\end{aligned}
$$

3.5. Pseudo Singular Points. Pseudo singular points are defined as singular points of the "normalized slow dynamics," that is, as the set of points for which we have

$$
\begin{aligned}
& \operatorname{det}\left[J_{\left(y_{1}, y_{2}\right)}\right]=0 \\
& \left(\frac{\partial g_{1}}{\partial x_{1}} \dot{x}_{1}+\frac{\partial g_{1}}{\partial x_{2}} \dot{x}_{2}\right) \frac{\partial g_{2}}{\partial y_{2}}-\left(\frac{\partial g_{2}}{\partial x_{1}} \dot{x}_{1}+\frac{\partial g_{2}}{\partial x_{2}} \dot{x}_{2}\right) \frac{\partial g_{1}}{\partial y_{2}} \\
& \quad=0 \\
& \left(\frac{\partial g_{1}}{\partial x_{1}} \dot{x}_{1}+\frac{\partial g_{1}}{\partial x_{2}} \dot{x}_{2}\right) \frac{\partial g_{2}}{\partial y_{1}}-\left(\frac{\partial g_{2}}{\partial x_{1}} \dot{x}_{1}+\frac{\partial g_{2}}{\partial x_{2}} \dot{x}_{2}\right) \frac{\partial g_{1}}{\partial y_{1}} \\
& \quad=0, \\
& g_{1}\left(x_{1}, x_{2}, y_{1}, y_{2}\right)=0 \\
& g_{2}\left(x_{1}, x_{2}, y_{1}, y_{2}\right)=0 .
\end{aligned}
$$

Remark 1. Let us notice on one hand that (22b) and (22c) are linearly dependent and on the other hand that contrary to previous works we do not use the "desingularized vector field" (21) but the "normalized slow dynamics" (20). 
The Jacobian matrix of system (20) reads

$$
J_{\left(F_{1}, F_{2}, G_{1}, G_{2}\right)}=\left(\begin{array}{cccc}
\frac{\partial F_{1}}{\partial x_{1}} & \frac{\partial F_{1}}{\partial x_{2}} & \frac{\partial F_{1}}{\partial y_{1}} & \frac{\partial F_{1}}{\partial y_{2}} \\
\frac{\partial F_{2}}{\partial x_{1}} & \frac{\partial F_{2}}{\partial x_{2}} & \frac{\partial F_{2}}{\partial y_{1}} & \frac{\partial F_{2}}{\partial y_{2}} \\
\frac{\partial G_{1}}{\partial x_{1}} & \frac{\partial G_{1}}{\partial x_{2}} & \frac{\partial G_{1}}{\partial y_{1}} & \frac{\partial G_{1}}{\partial y_{2}} \\
\frac{\partial G_{2}}{\partial x_{1}} & \frac{\partial G_{2}}{\partial x_{2}} & \frac{\partial G_{2}}{\partial y_{1}} & \frac{\partial G_{2}}{\partial y_{2}}
\end{array}\right)
$$

3.6. Extension of Benoît's Generic Hypothesis. Without loss of generality, it seems reasonable to extend Benoit's generic hypotheses introduced for the three-dimensional case to the four-dimensional case. So, first, let us suppose that by a "standard translation" the pseudo singular point can be shifted at the origin $O(0,0,0,0)$ and that by a "standard rotation" of $y_{1}$-axis that the slow manifold is tangent to $\left(x_{2}, x_{3}, y_{1}\right)$ hyperplane, so we have

$$
\begin{aligned}
& f_{1}(0,0,0,0)=g_{1}(0,0,0,0)=0 \\
& \left.\frac{\partial g_{1}}{\partial x_{2}}\right|_{(0,0,0,0)}=\left.\frac{\partial g_{1}}{\partial x_{3}}\right|_{(0,0,0,0)}=\left.\frac{\partial g_{1}}{\partial y_{1}}\right|_{(0,0,0,0)}=0 .
\end{aligned}
$$

Then, let us make the following assumptions for the nondegeneracy of the folded singularity:

$$
\begin{aligned}
& f_{2}(0,0,0,0) \neq 0 \\
& \left.\frac{\partial g_{1}}{\partial x_{1}}\right|_{(0,0,0,0)} \neq 0 ; \\
& \left.\frac{\partial^{2} g_{1}}{\partial y_{1}^{2}}\right|_{(0,0,0,0)} \neq 0 .
\end{aligned}
$$

According to these generic hypotheses ((24)-(25)), the Jacobian matrix (23) reads

$$
\begin{aligned}
& J_{\left(F_{1}, F_{2}, G_{1}, G_{2}\right)} \\
& =\left(\begin{array}{cccc}
0 & 0 & 0 & 0 \\
-f_{2} \frac{\partial P}{\partial x_{1}} & -f_{2} \frac{\partial P}{\partial x_{2}} & -f_{2} \frac{\partial P}{\partial y_{1}} & -f_{2} \frac{\partial P}{\partial y_{2}} \\
a_{31} & a_{32} & a_{33} & a_{34} \\
a_{41} & a_{42} & a_{43} & a_{44}
\end{array}\right),
\end{aligned}
$$

where

$$
\begin{aligned}
& P=\operatorname{det}\left[J_{\left(y_{1}, y_{2}\right)}\right] \text {, } \\
& a_{3 i}=-f_{2} \frac{\partial g_{2}}{\partial x_{2}} \frac{\partial^{2} g_{1}}{\partial y_{2} \partial x_{i}}+\frac{\partial g_{2}}{\partial y_{2}}\left(f_{2} \frac{\partial^{2} g_{1}}{\partial x_{2} \partial x_{i}}+\frac{\partial g_{1}}{\partial x_{1}} \frac{\partial f_{1}}{\partial x_{i}}\right) \\
& \text { for } i=1,2 \text {, } \\
& a_{3 i}=-f_{2} \frac{\partial g_{2}}{\partial x_{2}} \frac{\partial^{2} g_{1}}{\partial y_{2} \partial y_{i}}+\frac{\partial g_{2}}{\partial y_{2}}\left(f_{2} \frac{\partial^{2} g_{1}}{\partial x_{2} \partial y_{i}}+\frac{\partial g_{1}}{\partial x_{1}} \frac{\partial f_{1}}{\partial y_{i}}\right) \\
& \text { for } i=3,4 \text {, } \\
& a_{4 i}=f_{2} \frac{\partial g_{2}}{\partial x_{2}} \frac{\partial^{2} g_{1}}{\partial y_{1} \partial x_{i}}-\frac{\partial g_{2}}{\partial y_{1}}\left(f_{2} \frac{\partial^{2} g_{1}}{\partial x_{2} \partial x_{i}}+\frac{\partial g_{1}}{\partial x_{1}} \frac{\partial f_{1}}{\partial x_{i}}\right) \\
& \text { for } i=1,2 \text {, } \\
& a_{4 i}=f_{2} \frac{\partial g_{2}}{\partial x_{2}} \frac{\partial^{2} g_{1}}{\partial y_{1} \partial y_{i}}-\frac{\partial g_{2}}{\partial y_{1}}\left(f_{2} \frac{\partial^{2} g_{1}}{\partial x_{2} \partial y_{i}}+\frac{\partial g_{1}}{\partial x_{1}} \frac{\partial f_{1}}{\partial y_{i}}\right) \\
& \text { for } i=3,4 \text {. }
\end{aligned}
$$

Thus, we have the following Cayley-Hamilton eigenpolynomial associated with such Jacobian matrix (26) evaluated at the pseudo singular point, that is, at the origin:

$$
\lambda^{4}-\sigma_{1} \lambda^{3}+\sigma_{2} \lambda^{2}-\sigma_{3} \lambda+\sigma_{4}=0,
$$

where $\sigma_{1}=\operatorname{Tr}(J)$ is the sum of all first-order diagonal minors of $J$, that is, the trace of the Jacobian matrix $J, \sigma_{2}$ represents the sum of all second-order diagonal minors of $J$, and $\sigma_{3}$ represents the sum of all third-order diagonal minors of $J$. It appears that $\sigma_{4}=|J|=0$ since one row of the Jacobian matrix (26) is null. So, the eigenpolynomial reduces to

$$
\lambda\left(\lambda^{3}-\sigma_{1} \lambda^{2}+\sigma_{2} \lambda-\sigma_{3}\right)=0 .
$$

But, according to Wechselberger [11], $\sigma_{3}$ vanishes at a pseudo singular point as it is easy to prove it. So, the eigenpolynomial (29) is reduced to

$$
\lambda^{2}\left(\lambda^{2}-\sigma_{1} \lambda+\sigma_{2}\right)=0 .
$$

Let $\lambda_{i}$ be the eigenvalues of eigenpolynomial (30) and let us denote by $\lambda_{3,4}=0$ the obvious double root of this polynomial. We have

$$
\begin{aligned}
\sigma_{1} & =\operatorname{Tr}\left(J_{\left(F_{1}, F_{2}, G_{1}, G_{2}\right)}\right)=\lambda_{1}+\lambda_{2}=\frac{\partial g_{2}}{\partial x_{1}} \frac{\partial g_{1}}{\partial y_{1}} \frac{\partial f_{1}}{\partial y_{2}}, \\
\sigma_{2} & =\sum_{i=1}^{3} \mid J_{\left(F_{1}, F_{2}, G_{1}, G_{2}\right)}^{i i}=\lambda_{1} \lambda_{2}=\left(\frac{\partial g_{1}}{\partial y_{1}}\right)^{2} \\
& .\left[f_{2}^{2}\left(\frac{\partial^{2} g_{2}}{\partial x_{2}^{2}} \frac{\partial^{2} g_{2}}{\partial y_{2}^{2}}-\left(\frac{\partial^{2} g_{2}}{\partial x_{2} \partial y_{2}}\right)^{2}\right)\right. \\
& \left.+f_{2} \frac{\partial g_{2}}{\partial x_{1}}\left(\frac{\partial^{2} g_{2}}{\partial y_{2}^{2}} \frac{\partial f_{1}}{\partial x_{2}}-\frac{\partial^{2} g_{2}}{\partial x_{2} \partial y_{2}} \frac{\partial f_{1}}{\partial y_{2}}\right)\right],
\end{aligned}
$$


where $\sigma_{1}=\operatorname{Tr}\left(J_{\left(F_{1}, F_{2}, G_{1}, G_{2}\right)}\right)=p$ is is the sum of all firstorder diagonal minors of $J_{\left(F_{1}, F_{2}, G_{1}, G_{2}\right)}$, that is, the trace of the Jacobian matrix $J_{\left(F_{1}, F_{2}, G_{1}, G_{2}\right)}$, and $\sigma_{2}=\sum_{i=1}^{3}\left|J_{\left(F_{1}, F_{2}, G_{1}, G_{2}\right)}^{i i}\right|=q$ represents the sum of all second-order diagonal minors of $J_{\left(F_{1}, F_{2}, G_{1}, G_{2}\right)}$. Thus, the pseudo singular point is of saddle-type iff the following conditions $C_{1}$ and $C_{2}$ are verified:

$$
\begin{aligned}
& C_{1}: \Delta=p^{2}-4 q>0, \\
& C_{2}: q<0 .
\end{aligned}
$$

Condition $C_{1}$ is systematically satisfied provided that condition $C_{2}$ is verified. Thus, the pseudo singular point is of saddle-type iff $q<0$.

3.7. Canard Existence in $\mathbb{R}^{2+2}$. Following the works of Wechselberger [11] it can be stated, while using a standard polynomial change of variables, that any $n$-dimensional singularly perturbed systems with $k$ slow variables $(k \geqslant 2)$ and $m$ fast $(m \geqslant 1)(1)$ can be transformed into the following "normal form":

$$
\begin{aligned}
\dot{x_{1}}= & \tilde{a} x_{2}+\tilde{b} y_{2}+O\left(x_{1}, \epsilon, x_{2}^{2}, x_{2} y_{2}, y_{2}^{2}\right), \\
\dot{x_{2}}= & 1+O\left(x_{1}, x_{2}, y_{2}, \epsilon\right), \\
\epsilon \dot{y}_{1}= & \widetilde{c} y_{1}+O\left(\epsilon x_{1}, \epsilon x_{2}, \epsilon y_{2}, x_{1}^{2}, x_{2}^{2}, y_{2}^{2}, x_{2} y_{2}\right), \\
\epsilon \dot{y}_{2}= & -\left(x_{1}+y_{2}^{2}\right) \\
& +O\left(\epsilon x_{1}, \epsilon x_{2}, \epsilon y_{2}, \epsilon^{2}, x_{1}^{2} y_{2}, y_{2}^{3}, x_{1} x_{2} y_{2}\right) .
\end{aligned}
$$

We establish in the Appendix for any four-dimensional singularly perturbed systems (13a), (13b), (13c), and (13d) with $k=2$ slow and $m=2$ fast variables that

$$
\begin{aligned}
\widetilde{a} & =\frac{1}{2}\left[f_{2}^{2}\left(\frac{\partial^{2} g_{2}}{\partial x_{2}^{2}} \frac{\partial^{2} g_{2}}{\partial y_{2}^{2}}-\left(\frac{\partial^{2} g_{2}}{\partial x_{2} \partial y_{2}}\right)^{2}\right)\right. \\
& \left.+f_{2} \frac{\partial g_{2}}{\partial x_{1}}\left(\frac{\partial^{2} g_{2}}{\partial y_{2}^{2}} \frac{\partial f_{1}}{\partial x_{2}}-\frac{\partial^{2} g_{2}}{\partial x_{2} \partial y_{2}} \frac{\partial f_{1}}{\partial y_{2}}\right)\right], \\
\tilde{b}= & -\frac{\partial g_{2}}{\partial x_{1}} \frac{\partial f_{1}}{\partial y_{2}} \\
\tilde{c} & =\frac{\partial g_{1}}{\partial y_{1}} .
\end{aligned}
$$

Thus, in his paper Wechselberger [11, p. 3304] provided in the framework of "standard analysis" a generalization of Benoît's theorem [2] for any $n$-dimensional singularly perturbed systems with $k$ slow variables $(k \geqslant 2)$ and $m$ fast $(m \geqslant 1)$. According to his theorem presented below as Theorem 2 he proved the existence of canard solutions for the original system (1).

Theorem 2. In the folded saddle case of system (33) singular canards perturb to maximal canards solutions for sufficiently small $\varepsilon \ll 1$.
Proof. See Wechselberger [11].

Since our method does not use the "desingularized vector field" (21) but the "normalized slow dynamics" (20), we have the following proposition.

Proposition 3. If the normalized slow dynamics (20) has a pseudo singular point of saddle-type, that is, if the sum $\sigma_{2}$ of all second-order diagonal minors of the Jacobian matrix of the normalized slow dynamics (20) evaluated at the pseudo singular point is negative, that is, if $\sigma_{2}<0$ then, according to Theorem 2, system (13a), (13b), (13c), and (13d) exhibits a canard solution which evolves from the attractive part of the slow manifold towards its repelling part.

Proof. By making some smooth changes of time and smooth changes of coordinates (see the Appendix) we brought system (13a), (13b), (13c), and (13d) to the following "normal form":

$$
\begin{aligned}
\dot{x}_{1}= & \widetilde{a} x_{2}+\widetilde{b} y_{2}+O\left(x_{1}, \epsilon, x_{2}^{2}, x_{2} y_{2}, y_{2}^{2}\right), \\
\dot{x_{2}}= & 1+O\left(x_{1}, x_{2}, y_{2}, \epsilon\right), \\
\epsilon \dot{y}_{1}= & \widetilde{c} y_{1}+O\left(\epsilon x_{1}, \epsilon x_{2}, \epsilon y_{2}, x_{1}^{2}, x_{2}^{2}, y_{2}^{2}, x_{2} y_{2}\right), \\
\epsilon \dot{y}_{2}= & -\left(x_{1}+y_{2}^{2}\right) \\
& +O\left(\epsilon x_{1}, \epsilon x_{2}, \epsilon y_{2}, \epsilon^{2}, x_{1}^{2} y_{2}, y_{2}^{3}, x_{1} x_{2} y_{2}\right) .
\end{aligned}
$$

Then, we deduce that the condition for the pseudo singular point to be of saddle-type is $\tilde{a}<0$. According to (32) it is easy to verify that

$$
\begin{aligned}
& \sigma_{1}=\operatorname{Tr}\left(J_{\left(F_{1}, F_{2}, G_{1}, G_{2}\right)}\right)=\lambda_{1}+\lambda_{2}=-\widetilde{b} \widetilde{c}, \\
& \sigma_{2}=\sum_{i=1}^{3}\left|J_{\left(F_{1}, F_{2}, G_{1}, G_{2}\right)}^{i i}\right|=\lambda_{1} \lambda_{2}=2 \widetilde{a} \widetilde{c}^{2} .
\end{aligned}
$$

So, the condition for which the pseudo singular point is of saddle-type, that is, $\sigma_{2}<0$, is identical to that proposed by Wechselberger [11, p. 3298] in his theorem; that is, $\tilde{a}<0$.

So, Proposition 3 can be used to state the existence of canard solution for such systems. Application of Proposition 3 to the coupled FitzHugh-Nagumo equations, presented in Section 4, which is a four-dimensional singularly perturbed system with two slow and two fast variables will enable proving, as many previous works such as those of Tchizawa and Campbell [18] and Tchizawa [19-24], the existence of "canard solutions" in such system. According to Tchizawa [25], it is very important to notice on one hand that the fast equation has 2 dimensions in the system $\mathbb{R}^{2+2}$ and on the other hand that the fast system can give attractive, repulsive, or attractive-repulsive part at each pseudo singular point. Then, Tchizawa [25] has established that the jumping direction can be shown using the eigenvectors. In the same way we will find again the results of Rubin and Wechselberger [26] concerning the existence of "canard solutions" in the Hodgkin-Huxley model but with a set of more realistic parameters used in Chua et al. [27, 28]. 


\section{Coupled FitzHugh-Nagumo Equations}

The FitzHugh-Nagumo model $[29,30]$ is a simplified version of the Hodgkin-Huxley model [31] which models in a detailed manner activation and deactivation dynamics of a spiking neuron. By coupling two FitzHugh-Nagumo models Tchizawa and Campbell [18] and Tchizawa [19, 24] obtained the following four-dimensional singularly perturbed system with two slow and two fast variables:

$$
\begin{aligned}
\frac{d x_{1}}{d t} & =\frac{1}{c}\left(y_{1}+b x_{1}\right), \\
\frac{d x_{2}}{d t} & =\frac{1}{c}\left(y_{2}+b x_{2}\right), \\
\varepsilon \frac{d y_{1}}{d t} & =x_{1}-\frac{y_{1}^{3}}{3}+y_{2}, \\
\varepsilon \frac{d y_{2}}{d t} & =x_{2}-\frac{y_{2}^{3}}{3}+y_{1},
\end{aligned}
$$

where $0<\varepsilon \ll 1$ and $b$ is the "canard parameter" or "duck parameter" while $c$ is a scale factor.

4.1. Slow Manifold and Constrained System. The slow manifold equation of system (37a), (37b), (37c), and (37d) is defined by setting $\varepsilon=0$ in (37c) and (37d). Thus, we obtain

$$
\begin{aligned}
\frac{d x_{1}}{d t} & =\frac{1}{c}\left(y_{1}+b x_{1}\right) \\
\frac{d x_{2}}{d t} & =\frac{1}{c}\left(y_{2}+b x_{2}\right) \\
\frac{d y_{1}}{d t} & =-\frac{(1 / c)\left(y_{2}+b x_{2}\right)+\left(y_{2}^{2} / c\right)\left(y_{1}+b x_{1}\right)}{y_{1}^{2} y_{2}^{2}-1}, \\
\frac{d y_{2}}{d t} & =-\frac{(1 / c)\left(y_{1}+b x_{1}\right)+\left(y_{1}^{2} / c\right)\left(y_{2}+b x_{2}\right)}{y_{1}^{2} y_{2}^{2}-1}, \\
0 & =x_{1}-\frac{y_{1}^{3}}{3}+y_{2}, \\
0 & =x_{2}-\frac{y_{2}^{3}}{3}+y_{1} .
\end{aligned}
$$

4.2. Normalized Slow Dynamics. Then, by rescaling the time by setting $t=-\operatorname{det}\left[J_{\left(y_{1}, y_{2}\right)}\right] \tau=-\left(y_{1}^{2} y_{2}^{2}-1\right)$ we obtain the "normalized slow dynamics":

$$
\begin{aligned}
\frac{d x_{1}}{d t} & =-\frac{1}{c}\left(y_{1}+b x_{1}\right)\left(y_{1}^{2} y_{2}^{2}-1\right) \\
& =F_{1}\left(x_{1}, x_{2}, y_{1}, y_{2}\right) \\
\frac{d x_{2}}{d t} & =-\frac{1}{c}\left(y_{2}+b x_{2}\right)\left(y_{1}^{2} y_{2}^{2}-1\right) \\
& =F_{2}\left(x_{1}, x_{2}, y_{1}, y_{2}\right),
\end{aligned}
$$

$$
\begin{aligned}
\frac{d y_{1}}{d t}= & \frac{1}{c}\left(y_{2}+b x_{2}\right)+\frac{y_{2}^{2}}{c}\left(y_{1}+b x_{1}\right) \\
= & G_{1}\left(x_{1}, x_{2}, y_{1}, y_{2}\right), \\
\frac{d y_{2}}{d t}= & \frac{1}{c}\left(y_{1}+b x_{1}\right) \\
& +\frac{y_{1}^{2}}{c}\left(y_{2}+b x_{2}\right) G_{2}\left(x_{1}, x_{2}, y_{1}, y_{2}\right), \\
0= & x_{1}-\frac{y_{1}^{3}}{3}+y_{2}, \\
0= & x_{2}-\frac{y_{2}^{3}}{3}+y_{1} .
\end{aligned}
$$

4.3. Pseudo Singular Points. From (22a), (22b), (22c), (22d), and (22e), the pseudo singular points of system (37a), (37b), (37c), and (37d) are defined by

$$
\begin{aligned}
& \operatorname{det}\left[J_{\left(y_{1}, y_{2}\right)}\right]=y_{1}^{2} y_{2}^{2}-1=0 \\
& \left(\frac{\partial g_{1}}{\partial x_{1}} \dot{x}_{1}+\frac{\partial g_{1}}{\partial x_{2}} \dot{x}_{2}\right) \frac{\partial g_{2}}{\partial y_{2}}-\left(\frac{\partial g_{2}}{\partial x_{1}} \dot{x}_{1}+\frac{\partial g_{2}}{\partial x_{2}} \dot{x}_{2}\right) \frac{\partial g_{1}}{\partial y_{2}} \\
& =\frac{1}{c}\left(y_{2}+b x_{2}\right)+\frac{y_{2}^{2}}{c}\left(y_{1}+b x_{1}\right)=0 \\
& \left(\frac{\partial g_{1}}{\partial x_{1}} \dot{x}_{1}+\frac{\partial g_{1}}{\partial x_{2}} \dot{x}_{2}\right) \frac{\partial g_{2}}{\partial y_{1}}-\left(\frac{\partial g_{2}}{\partial x_{1}} \dot{x}_{1}+\frac{\partial g_{2}}{\partial x_{2}} \dot{x}_{2}\right) \frac{\partial g_{1}}{\partial y_{1}} \\
& =\frac{1}{c}\left(y_{1}+b x_{1}\right)+\frac{y_{1}^{2}}{c}\left(y_{2}+b x_{2}\right)=0 \\
& g_{1}\left(x_{1}, x_{2}, y_{1}, y_{2}\right)=x_{1}-\frac{y_{1}^{3}}{3}+y_{2}=0 \\
& g_{2}\left(x_{1}, x_{2}, y_{1}, y_{2}\right)=x_{2}-\frac{y_{2}^{3}}{3}+y_{1}=0 .
\end{aligned}
$$

According to Tchizawa and Campbell [18] and Tchizawa $[19,20]$, there are six pseudo singular points with the last four depending on the parameter $b$ :

$$
\begin{aligned}
& \left(\tilde{x}_{1}, \tilde{x}_{2}, \tilde{y}_{1}, \tilde{y}_{2}\right)=\left( \pm \frac{4}{3}, \mp \frac{4}{3}, \pm 1, \mp 1\right), \\
& \left(\tilde{x}_{1}, \tilde{x}_{2}, \tilde{y}_{1}, \tilde{y}_{2}\right) \\
& =\left( \pm \frac{\sqrt{\left(3-\sqrt{9-4 b^{2}}\right) / b}\left(3+2 \sqrt{9-4 b^{2}}\right)}{3 \sqrt{2} b},\right. \\
& \mp \frac{\sqrt{\left(3-\sqrt{9-4 b^{2}}\right) / b}\left(9-8 b^{2}+3 \sqrt{9-4 b^{2}}\right)}{6 \sqrt{2} b^{2}}, \\
& \left.\mp \sqrt{\frac{3-\sqrt{9-4 b^{2}}}{2 b}, \mp \frac{\sqrt{2 b}}{\sqrt{3-\sqrt{9-4 b^{2}}}}}\right),
\end{aligned}
$$




$$
\begin{aligned}
& \left(\tilde{x}_{1}, \tilde{x}_{2}, \tilde{y}_{1}, \tilde{y}_{2}\right) \\
& =\left( \pm \frac{\left(3-2 \sqrt{9-4 b^{2}}\right) \sqrt{\left(3+\sqrt{9-4 b^{2}}\right) / b}}{3 \sqrt{2} b},\right. \\
& \mp \frac{\sqrt{\left(3+\sqrt{9-4 b^{2}}\right) / b}\left(9-8 b^{2}-3 \sqrt{9-4 b^{2}}\right)}{6 \sqrt{2} b^{2}}, \\
& \left.\mp \sqrt{\frac{3-\sqrt{9-4 b^{2}}}{2 b}}, \mp \frac{\sqrt{2 b}}{\sqrt{3-\sqrt{9-4 b^{2}}}}\right) .
\end{aligned}
$$

4.4. Canard Existence in Coupled FitzHugh-Nagumo Equations. The Jacobian matrix of system (39) evaluated at the pseudo singular points (41a) reads

$$
\begin{aligned}
& J_{\left(F_{1}, F_{2}, G_{1}, G_{2}\right)} \\
& =\left(\begin{array}{cccc}
0 & 0 & \frac{2(3+4 b)}{3 c} & -\frac{2(3+4 b)}{3 c} \\
0 & 0 & -\frac{2(3+4 b)}{3 c} & \frac{2(3+4 b)}{3 c} \\
\frac{b}{c} & \frac{b}{c} & \frac{1}{c} & -\frac{3+8 b}{3 c} \\
\frac{b}{c} & \frac{b}{c} & -\frac{3+8 b}{3 c} & \frac{1}{c}
\end{array}\right) .
\end{aligned}
$$

Remark 4. Although the pseudo singular points have not been shifted at the origin extension of Benoît's generic hypotheses (24)-(25) are satisfied. In other words, we have $\sigma_{4}=\sigma_{3}=0$.
According to (31) we find that

$$
\begin{aligned}
& p=\sigma_{1}=\operatorname{Tr}(J)=+\frac{2}{c}, \\
& q=\sigma_{2}=-\frac{16 b(3+4 b)}{9 c^{2}} .
\end{aligned}
$$

Thus, according to Proposition 3, the pseudo singular points are of saddle-type if and only if:

$$
-\frac{16 b(3+4 b)}{9 c^{2}}<0 .
$$

So, we have conditions $C_{1}$ and $C_{2}$ :

$$
\begin{aligned}
& C_{1}: \Delta=\frac{4(3+8 b)^{2}}{9 c^{2}}>0, \\
& C_{2}: q=-\frac{16 b(3+4 b)}{9 c^{2}}<0 .
\end{aligned}
$$

Let us choose arbitrarily $b$ as the "canard parameter" or "duck parameter." Obviously, it appears that the condition $C_{1}$ is still satisfied. Finally, the pseudo singular points are of saddle-type if and only if we have

$$
\begin{aligned}
& b>0 \text { or } \\
& b<-\frac{3}{4} .
\end{aligned}
$$

Remark 5. Let us notice that the pseudo singular points are of node-type if $-3 / 4<b<0$ as stated by Tchizawa and Campbell [18] and Tchizawa [19, 20].

The Jacobian matrix $J_{\left(F_{1}, F_{2}, G_{1}, G_{2}\right)}$ of system (39) evaluated at the pseudo singular points (41b) reads

$$
\left(\begin{array}{cccc}
0 & 0 & -\frac{4 \sqrt{9-4 b^{2}}}{3 c} & -\frac{2\left(-9+4 b^{2}+3 \sqrt{9-4 b^{2}}\right)}{3 b c} \\
0 & 0 & \frac{2\left(-9+4 b^{2}+3 \sqrt{9-4 b^{2}}\right)}{3 b c} & \frac{4 \sqrt{9-4 b^{2}}}{3 c} \\
\frac{3+\sqrt{9-4 b^{2}}}{2 c} & \frac{b}{c} & \frac{3+\sqrt{9-4 b^{2}}}{2 b c} & \frac{3-4 \sqrt{9-4 b^{2}}}{3 c} \\
\frac{b}{c} & \frac{3-\sqrt{9-4 b^{2}}}{2 c} & \frac{3+4 \sqrt{9-4 b^{2}}}{3 c} & \frac{3-\sqrt{9-4 b^{2}}}{2 b c}
\end{array}\right) .
$$

Remark 6. Although the pseudo singular points have not been shifted at the origin extension of Benoît's generic hypotheses (24)-(25) are satisfied. In other words, we have $\sigma_{4}=\sigma_{3}=0$.

According to (31) we find that

$$
\begin{aligned}
& p=\sigma_{1}=\operatorname{Tr}(J)=+\frac{3}{b c}, \\
& q=\sigma_{2}=\frac{16\left(9-4 b^{2}\right)}{9 c^{2}} .
\end{aligned}
$$

Thus, according to Proposition 3, the pseudo singular points are of saddle-type if and only if 
So, we have conditions $C_{1}$ and $C_{2}$ :

$$
\begin{aligned}
& C_{1}: \Delta=\left(\frac{3}{b c}\right)^{2}-\frac{64\left(9-4 b^{2}\right)}{9 c^{2}}>0, \\
& C_{2}: q=\frac{16\left(9-4 b^{2}\right)}{9 c^{2}}<0 .
\end{aligned}
$$

Let us choose arbitrarily $b$ as the "canard parameter" or "duck parameter." Obviously, it appears that if the condition $C_{2}$ is verified, then the condition $C_{1}$ is de facto satisfied. Finally, the pseudo singular points are of saddle-type if and only if we have

$$
\begin{aligned}
& b>\frac{3}{2} \text { or } \\
& b<-\frac{3}{2} .
\end{aligned}
$$

Remark 7. Because of the symmetry of these coupled FitzHugh-Nagumo equations, the Jacobian matrix of system (39) evaluated at the pseudo singular points (41c) provides the same result as just above.

\section{Hodgkin-Huxley Model}

The original Hodgkin-Huxley model [31] is described by the following system of four nonlinear ordinary differential equations:

$$
\begin{aligned}
\frac{d V}{d t} & =\frac{1}{C_{\mathrm{M}}}\left[I-\bar{g}_{\mathrm{K}} n^{4}\left(V-V_{\mathrm{K}}\right)-\bar{g}_{\mathrm{Na}} m^{3} h\left(V-V_{\mathrm{Na}}\right)\right. \\
& \left.-\bar{g}_{\mathrm{L}}\left(V-V_{\mathrm{L}}\right)\right], \\
\frac{d n}{d t} & =\alpha_{n}(V)(1-n)-\beta_{n}(V) n, \\
\frac{d m}{d t} & =\alpha_{m}(V)(1-m)-\beta_{m}(V) m, \\
\frac{d h}{d t} & =\alpha_{h}(V)(1-h)-\beta_{h}(V) h,
\end{aligned}
$$

where:

$$
\begin{aligned}
& \alpha_{n}(V)=\frac{0.01(V+10)}{(\exp ((V+10) / 10)-1)}, \\
& \beta_{n}(V)=0.125 \exp \left(\frac{V}{80}\right) \\
& \alpha_{m}(V)=\frac{0.1(V+25)}{(\exp ((V+25) / 10)-1)} \\
& \beta_{m}(V)=4 \exp \left(\frac{V}{18}\right) \\
& \alpha_{h}(V)=0.07 \exp \left(\frac{V}{20}\right) \\
& \beta_{n}(V)=\frac{1}{(\exp ((V+30) / 10)+1)}
\end{aligned}
$$

The first equation (52a) results from the application of Kirchhoff's law to the space clamped squid giant axon. Thus, the total membrane current $C_{\mathrm{M}} d V / d t$ for which $C_{\mathrm{M}}$ represents the specific membrane capacity and $V$ the displacement of the membrane potential from its resting value is equal to the sum of the following intrinsic currents:

$$
\begin{aligned}
I_{\mathrm{K}} & =\bar{g}_{\mathrm{K}} n^{4}\left(V-V_{\mathrm{K}}\right), \\
I_{\mathrm{Na}} & =\bar{g}_{\mathrm{Na}} m^{3} h\left(V-V_{\mathrm{Na}}\right), \\
I_{\mathrm{L}} & =\bar{g}_{\mathrm{L}}\left(V-V_{\mathrm{L}}\right),
\end{aligned}
$$

where $I_{\mathrm{K}}$ is a delayed rectifier potassium current, $I_{\mathrm{Na}}$ is fast sodium current, and $I_{\mathrm{L}}$ is the "leakage current." The parameter $I$ is the total membrane current density, inward positive, that is, the total current injected into the space clamped squid giant axon, and $V_{\mathrm{K}}, V_{\mathrm{Na}}$, and $V_{\mathrm{L}}$ are the equilibrium potentials of potassium, sodium, and "leakage current," respectively. The maximal specific conductance degrees of the ionic currents are denoted by $\bar{g}_{\mathrm{K}}, \bar{g}_{\mathrm{Na}}$, and $\bar{g}_{\mathrm{L}}$, respectively. Functions $\alpha_{n, m, h}$ and $\beta_{n, m, h}$ are gates' opening and closing rates depending on $V$. Variable $m$ denotes the activation of the sodium current, variable $h$ the inactivation of the sodium current, and variable $n$ the activation of the potassium current. These dimensionless gating variables vary in the range $[0,1]$.

Let us notice that the variables and symbols in ((52a), (52b), (52c), and (52d) and (53a), (53b), (53c), (53d), (53e), and (53f)) originally chosen by Hodgkin and Huxley and are different from those found in recent literatures, where the reference polarity of the voltage $V$ and the reference direction of the current $I$ are defined as the negative of the voltages and currents. We have opted to adopt the reference assumption in Hodgkin and Huxley [31] for ease in comparison of our results with those from Hodgkin and Huxley (for more details see Chua et al. $[27,28])$. The parameter values are exactly those chosen in the original Hodgkin-Huxley [31] works:

$$
\begin{aligned}
C_{\mathrm{M}} & =1.0 \mu \mathrm{F} / \mathrm{cm}^{2}, \\
V_{\mathrm{Na}} & =-115 \mathrm{mV}, \\
V_{\mathrm{K}} & =12 \mathrm{mV}, \\
V_{\mathrm{L}} & =-10.613 \mathrm{mV}, \\
\bar{g}_{\mathrm{Na}} & =120 \mathrm{mS} / \mathrm{cm}^{2}, \\
\bar{g}_{\mathrm{K}} & =36 \mathrm{mS} / \mathrm{cm}^{2}, \\
\bar{g}_{\mathrm{L}} & =0.3 \mathrm{mS} / \mathrm{cm}^{2} .
\end{aligned}
$$

According to Suckley and Biktashev [32] and Suckley [33], dimensionless functions $\bar{n}, \bar{h}$, and $\bar{m}$ called gates' instant equilibrium values, that is, steady-state relation for gating variables $n, h$, and $m$, respectively, as well as $\tau_{n}, \tau_{h}$, and $\tau_{m}$ called gates dynamics timescales in $\mathrm{ms}$, that is, time constant 
for gating variables $n, h$, and $m$, respectively, may be defined as follows:

$$
\begin{gathered}
\bar{n}(V)=\frac{\alpha_{n}(V)}{\alpha_{n}(V)+\beta_{n}(V)}, \\
\bar{h}(V)=\frac{\alpha_{h}(V)}{\alpha_{h}(V)+\beta_{h}(V)}, \\
\bar{m}(V)=\frac{\alpha_{m}(V)}{\alpha_{m}(V)+\beta_{m}(V)}, \\
\tau_{n}(V)=\frac{1}{\alpha_{n}(V)+\beta_{n}(V)}, \\
\tau_{h}(V)=\frac{1}{\alpha_{h}(V)+\beta_{h}(V)}, \\
\tau_{m}(V)=\frac{1}{\alpha_{m}(V)+\beta_{m}(V)} .
\end{gathered}
$$

By using (56a), (56b), (56c), (56d), (56e), and (56f), the original Hodgkin-Huxley model [31] reads

$$
\begin{aligned}
\frac{d V}{d t} & =\frac{1}{C_{\mathrm{M}}}\left[I-\bar{g}_{\mathrm{K}} n^{4}\left(V-V_{\mathrm{K}}\right)-\bar{g}_{\mathrm{Na}} m^{3} h\left(V-V_{\mathrm{Na}}\right)\right. \\
& \left.-\bar{g}_{\mathrm{L}}\left(V-V_{\mathrm{L}}\right)\right], \\
\frac{d n}{d t} & =\frac{\bar{n}-n}{\tau_{n}}, \\
\frac{d h}{d t} & =\frac{\bar{h}-h}{\tau_{h}}, \\
\frac{d m}{d t} & =\frac{\bar{m}-m}{\tau_{m}} .
\end{aligned}
$$

Now, in order to apply the singular perturbation method to the Hodgkin-Huxley model, two small multiplicative parameters $\varepsilon \ll 1$ are introduced. According to Suckley and Biktashev [32], Suckley [33], and Rubin and Wechselberger [26], the existence of two different timescales of evolution for couples of dynamic variables $(n, h)$ and $(m, V)$ enables justifying such an introduction. So, in order to differentiate slow variables from fast variables, Suckley and Biktashev [32], Suckley [33], and Rubin and Wechselberger [26] have plotted the inverse of "time constant for gating variable $i$," that is, $\tau_{i}^{-1}$ according to $V$ with $i=n, h, m$. In Figure 1 , they have been plotted for the original functions $\alpha_{i}$ and $\beta_{i}$ (53a). However, let us notice that this plot is exactly the same as those presented by Rubin and Wechselberger [26] (Figure 1) for a nondimensionalized three-dimensional Hodgkin-Huxley singularly perturbed system obtained after the following variable changes: $V \rightarrow-V$ and $\bar{I} \rightarrow-\bar{I}$; then $V \rightarrow V+65$ and finally $V \rightarrow V / 100$.

Figure 1 shows a plot of the functions $\tau_{i}^{-1}$ according to $V$ with $i=n, h, m$ over the physiological range. We observe that $\tau_{m}{ }^{-1}$ is of an order of magnitude bigger than $\tau_{h}{ }^{-1}$ and $\tau_{n}{ }^{-1}$, which are of comparable size. Indeed, we can deduce that the values of times scales are approximately $\tau_{m}{ }^{-1} \approx 10 \mathrm{~ms}^{-1}$ while

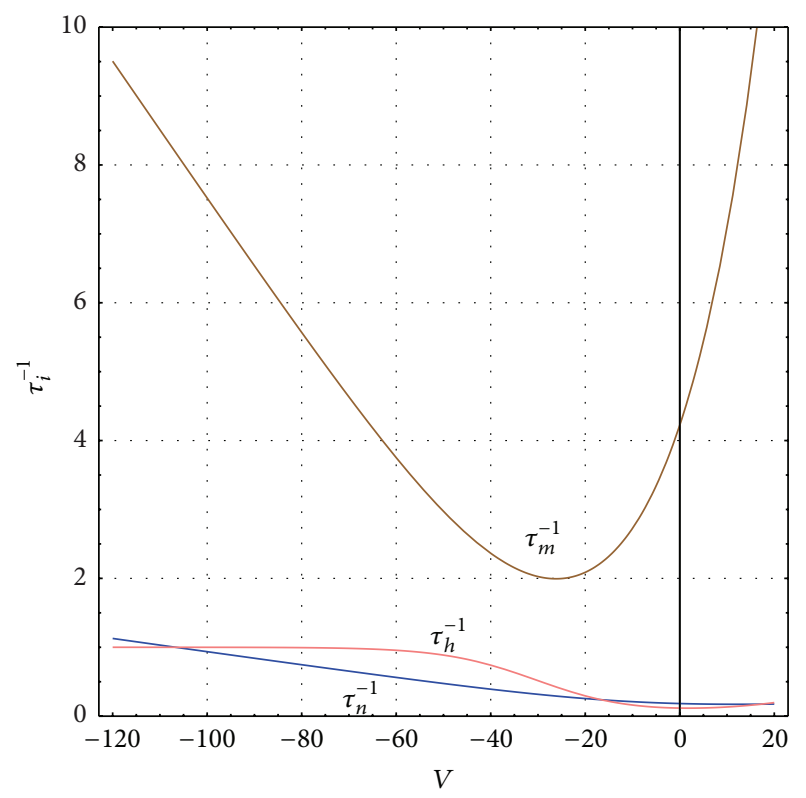

FIGURE 1: Graph of $1 / \tau_{i}\left(\mathrm{~ms}^{-1}\right)$ against $V(\mathrm{mV})$.

$\tau_{n}{ }^{-1} \approx \tau_{h}{ }^{-1} \approx 1 \mathrm{~ms}^{-1}$. Then, it appears that $m$ corresponds to the fast variable while $n$ and $h$ correspond to slow variables. Moreover, since the activation of the sodium channel $m$ is directly related to the dynamics of the membrane (action) potential $V$, Rubin and Wechselberger [26] consider that $m$ and $V$ evolve on the same fast timescale. So, the HodgkinHuxley model may be transformed into a singularly perturbed system with two timescales in which the slow variables are $n, h$ and the fast variables are $m, V$.

So, according to Awiszus et al. [34], Suckley and Biktashev [32], Suckley [33], and Rubin and Wechselberger [26] small multiplicative parameters $0<\varepsilon \ll 1$ in the original vector field of the Hodgkin-Huxley equations (57a), (57b), (57c), and (57d) may be identified while factorizing the right-hand side of (57a) by $\bar{g}_{\mathrm{Na}}$ and set:

$$
\begin{aligned}
& \bar{g}_{\mathrm{Na}} \longrightarrow \frac{\bar{g}_{\mathrm{Na}}}{\bar{g}_{\mathrm{Na}}}=1, \\
& \bar{g}_{\mathrm{K}} \longrightarrow \frac{\bar{g}_{\mathrm{K}}}{\bar{g}_{\mathrm{Na}}}=0.3, \\
& \bar{g}_{\mathrm{L}} \longrightarrow \frac{\bar{g}_{\mathrm{L}}}{\bar{g}_{\mathrm{Na}}}=0.0025 .
\end{aligned}
$$

Other parameters are kept as for the original HodgkinHuxley model [31]:

$$
\begin{aligned}
& C_{\mathrm{M}}=1.0 \mu \mathrm{F} / \mathrm{cm}^{2}, \\
& \bar{V}_{\mathrm{Na}}=-115 \mathrm{mV}, \\
& \bar{V}_{\mathrm{K}}=12 \mathrm{mV}, \\
& \bar{V}_{\mathrm{L}}=-10.613 \mathrm{mV} .
\end{aligned}
$$


Then, by posing $\bar{I} \rightarrow \bar{I} / \bar{g}_{\mathrm{Na}}, \varepsilon=C_{\mathrm{M}} / \bar{g}_{\mathrm{Na}}=1 / 120$, and $(n, h, m, V)=\left(x_{1}, x_{2}, y_{1}, y_{2}\right)$ to be consistent with the notations of Section 3, we obtain

$$
\begin{aligned}
\frac{d x_{1}}{d t}= & \frac{\bar{x}_{1}-x_{1}}{\tau_{1}}=f_{1}\left(x_{1}, x_{2}, y_{1}, y_{2}\right), \\
\frac{d x_{2}}{d t}= & \frac{\bar{x}_{2}-x_{2}}{\tau_{2}}=f_{1}\left(x_{1}, x_{2}, y_{1}, y_{2}\right), \\
\varepsilon \frac{d y_{1}}{d t}= & \frac{\bar{y}_{1}-y_{1}}{\tau_{3}}=g_{1}\left(x_{1}, x_{2}, y_{1}, y_{2}\right), \\
\varepsilon \frac{d y_{2}}{d t}= & \bar{I}-\bar{g}_{\mathrm{K}} x_{1}^{4}\left(y_{2}-V_{\mathrm{K}}\right)-\bar{g}_{\mathrm{Na}} y_{1}^{3} x_{2}\left(y_{2}-V_{\mathrm{Na}}\right) \\
& -\bar{g}_{\mathrm{L}}\left(y_{2}-V_{\mathrm{L}}\right)=g_{2}\left(x_{1}, x_{2}, y_{1}, y_{2}\right),
\end{aligned}
$$

where $\left(\bar{x}_{1}, \bar{x}_{2}, \bar{y}_{1}\right)=(\bar{n}, \bar{h}, \bar{m})$ and $\tau_{1,2,3}=\tau_{n, h, m}$.

Let us notice that the multiplicative parameter $\varepsilon$ has been introduced artificially in (60c). This is due to the fact that it has been stated above that the timescale of variable $m$, that is, $y_{1}$, is tenth times greater than the timescale of variables $n$ and $h$, that is, of variables $x_{1}$ and $x_{2}$. Moreover, this parameter is identical to those used in (60d) since it has been also considered that $m$ and $V$, that is, $y_{1}$ and $y_{2}$, evolve on the same fast timescale.

According to the Geometric Singular Perturbation Theory, the zero-order approximation in $\varepsilon$ of the slow manifold associated with the Hodgkin-Huxley model (60a), (60b), (60c), and (60d) is obtained by posing $\varepsilon=0$ in (60c) and (60d). So, the slow manifold is given by

$$
\begin{aligned}
& x_{2}=\frac{\bar{I}-\bar{g}_{\mathrm{K}} x_{1}^{4}\left(y_{2}-V_{\mathrm{K}}\right)-\bar{g}_{\mathrm{L}}\left(y_{2}-V_{\mathrm{L}}\right)}{\bar{g}_{\mathrm{Na}} \bar{y}_{1}^{3}\left(y_{2}-V_{\mathrm{Na}}\right)}, \\
& y_{1}=\bar{y}_{1}\left(y_{2}\right) .
\end{aligned}
$$

Then, the fast foliation is within the planes $x_{1}=$ constant and $x_{2}=$ constant .

The fold curve is defined as the location of the points, where $g_{1}\left(x_{1}, x_{2}, y_{1}, y_{2}\right)=0, g_{2}\left(x_{1}, x_{2}, y_{1}, y_{2}\right)=0$, and $\operatorname{det}\left[J_{\left(g_{1}, g_{2}\right)}\right]=0$. For the Hodgkin-Huxley model (60a), (60b), $(60 \mathrm{c})$, and (60d), the fold curve is thus given by (61a) and (61b) and by the determinant of the Jacobian matrix of the following fast foliation:

$$
\begin{aligned}
\frac{d y_{1}}{d t}= & \frac{\bar{y}_{1}-y_{1}}{\tau_{3}}=g_{1}\left(x_{1}, x_{2}, y_{1}, y_{2}\right), \\
\frac{d y_{2}}{d t}= & \bar{I}-\bar{g}_{\mathrm{K}} x_{1}^{4}\left(y_{2}-V_{\mathrm{K}}\right)-\bar{g}_{\mathrm{Na}} y_{1}^{3} x_{2}\left(y_{2}-V_{\mathrm{Na}}\right) \\
& -\bar{g}_{\mathrm{L}}\left(y_{2}-V_{\mathrm{L}}\right)=g_{2}\left(x_{1}, x_{2}, y_{1}, y_{2}\right) .
\end{aligned}
$$
reads

The Jacobian matrix of the fast foliation (62a) and (62b)

$$
\begin{aligned}
& J_{\left(g_{1}, g_{2}\right)} \\
& =\left(\begin{array}{cc}
\frac{\bar{y}_{1}^{\prime} \tau_{3}-\tau_{3}^{\prime}\left(\overline{y_{1}}-y_{1}\right)}{\tau_{3}^{2}} & -\frac{1}{\tau_{3}} \\
-\left(\bar{g}_{\mathrm{K}} x^{4}+\bar{g}_{\mathrm{Na}} y_{1}^{3} x_{2}+\bar{g}_{\mathrm{L}}\right) & -3 \bar{g}_{\mathrm{Na}} y_{1}^{2} x_{2}\left(y_{2}-V_{\mathrm{Na}}\right)
\end{array}\right),
\end{aligned}
$$

where $(I)$ denotes the derivative with respect to $y_{2}$. Then, taking into account (61b), that is, $y_{1}=\bar{y}_{1}$, we have

$$
\begin{aligned}
& J_{\left(g_{1}, g_{2}\right)} \\
& =\left(\begin{array}{cc}
{\overline{y_{1}}}_{\tau_{3}}^{\prime} & -\frac{1}{\tau_{3}} \\
-\left(\bar{g}_{\mathrm{K}} x_{1}^{4}+\bar{g}_{\mathrm{Na}} \bar{y}_{1}^{3} x_{2}+\bar{g}_{\mathrm{L}}\right) & -3 \bar{g}_{\mathrm{Na}} \bar{y}_{1}^{2} x_{2}\left(y_{2}-V_{\mathrm{Na}}\right)
\end{array}\right) .
\end{aligned}
$$

So, the determinant of the Jacobian matrix of the fast foliation (62a) and (62b) is

$$
\begin{aligned}
& \operatorname{det}\left(J_{\left(g_{1}, g_{2}\right)}\right)=-\frac{1}{\tau_{3}}\left[\bar{g}_{\mathrm{K}} x_{1}^{4}+\bar{g}_{\mathrm{Na}} \bar{y}_{1}^{3} x_{2}+\bar{g}_{\mathrm{L}}\right. \\
& \left.\quad+3 \bar{g}_{\mathrm{Na}} \bar{y}_{1}^{\prime} \bar{y}_{1}^{2} x_{2}\left(y_{2}-V_{\mathrm{Na}}\right)\right] .
\end{aligned}
$$

Thus, the condition for the fold curve is $\operatorname{det}\left(J_{\left(g_{1}, g_{2}\right)}\right)=0$, which gives

$$
\bar{g}_{\mathrm{K}} x_{1}^{4}+\bar{g}_{\mathrm{Na}} \bar{y}_{1}^{3} x_{2}+\bar{g}_{\mathrm{L}}+3 \bar{g}_{\mathrm{Na}} \bar{y}_{1}^{\prime} \bar{y}_{1}^{2} x_{2}\left(y_{2}-V_{\mathrm{Na}}\right)=0 .
$$

Therefore

$$
x_{2}=-\frac{\bar{g}_{\mathrm{K}} x_{1}^{4}+\bar{g}_{\mathrm{L}}}{\bar{g}_{\mathrm{Na}} \bar{y}_{1}^{2}\left(\bar{y}_{1}+3{\overline{y_{1}}}^{\prime}\left(y_{2}-V_{\mathrm{Na}}\right)\right)} .
$$

By subtracting (61a) from (67) we obtain $x_{1}$

$$
x_{1}=x_{1 f}=\left[\frac{-\bar{I}\left[\bar{y}_{1}+3{\overline{y_{1}}}^{\prime}\left(y_{2}-V_{\mathrm{Na}}\right)\right]+\bar{g}_{\mathrm{L}}\left(V_{\mathrm{Na}}-V_{\mathrm{L}}\right) \bar{y}_{1}+3{\overline{y_{1}}}^{\prime}\left(y_{2}-V_{\mathrm{Na}}\right)\left(y_{2}-V_{\mathrm{L}}\right)}{\bar{g}_{\mathrm{K}}\left[\left(V_{\mathrm{K}}-V_{\mathrm{Na}}\right) \bar{y}_{1}-3{\overline{y_{1}}}^{\prime}\left(y_{2}-V_{\mathrm{Na}}\right)\left(y_{2}-V_{\mathrm{K}}\right)\right]}\right]^{1 / 4} .
$$

Plugging this value of $x_{1}(68)$ into (67) provides

$$
\begin{aligned}
& x_{2}=x_{2 f} \\
& =\frac{\bar{I}+\bar{g}_{\mathrm{L}}\left(V_{\mathrm{K}}-V_{\mathrm{L}}\right)}{\bar{g}_{\mathrm{Na}} \bar{y}_{1}^{2}\left[\left(V_{\mathrm{Na}}-V_{\mathrm{K}}\right) \bar{y}_{1}+3 \bar{y}_{1}^{\prime}\left(y_{2}-V_{\mathrm{Na}}\right)\left(y_{2}-V_{\mathrm{K}}\right)\right]} .
\end{aligned}
$$

So, the fold curve is given by the set of parametric equations ((68)-(69)) in terms of $y_{2}$.

The pseudo singular points are given by (22a), (22b), (22c), (22d), and (22e) which read for the Hodgkin-Huxley model (60a), (60b), (60c), and (60d):

$$
\frac{\bar{y}_{1}-y_{1}}{\tau_{3}}=0 \text {, }
$$




$$
\begin{aligned}
& \bar{I}-\bar{g}_{\mathrm{K}} x_{1}^{4}\left(y_{2}-V_{\mathrm{K}}\right)-\bar{g}_{\mathrm{Na}} y_{1}^{3} x_{2}\left(y_{2}-V_{\mathrm{Na}}\right)-\bar{g}_{\mathrm{L}}\left(y_{2}\right. \\
& \left.-V_{\mathrm{L}}\right)=0, \\
& {\left[\frac{4 \bar{g}_{\mathrm{K}} x_{1}^{3}\left(y_{2}-V_{\mathrm{K}}\right)\left(x_{1}-\bar{x}_{1}\right)}{\tau_{1}}\right.} \\
& \left.\quad+\frac{\bar{g}_{\mathrm{Na}} y_{1}^{3}\left(y_{2}-V_{\mathrm{Na}}\right)\left(x_{2}-\bar{x}_{2}\right)}{\tau_{2}}\right]=0, \\
& {\left[\frac{4 \bar{g}_{\mathrm{K}} x_{1}^{3}\left(y_{2}-V_{\mathrm{K}}\right)\left(x_{1}-\bar{x}_{1}\right)}{\tau_{1}}\right] \frac{\bar{g}_{\mathrm{Na}} y_{1}^{3}\left(y_{2}-V_{\mathrm{Na}}\right)\left(x_{2}-\bar{x}_{2}\right)}{\tau_{2}}=0,} \\
& \quad \tau_{3}\left(\bar{g}_{\mathrm{K}} x_{1}^{4}+\bar{g}_{\mathrm{Na}} y_{1}^{3} x_{2}+\bar{g}_{\mathrm{L}}\right)+3 \bar{g}_{\mathrm{Na}} y_{1}^{2} x_{2}\left(y_{2}-V_{\mathrm{Na}}\right) \\
& \quad \cdot\left(\tau_{3} y_{1}^{\prime}+\left(y_{1}-\bar{y}_{1}\right) \tau_{3}^{\prime}\right)=0 .
\end{aligned}
$$

Let us notice that (70c) and (70d) are identical. Moreover, the definition of $\tau_{3}$ (56f) enables simplifying system (70a), (70b), (70c), (70d), and (70e). Thus, we have

$$
\begin{aligned}
& \bar{I}-\bar{g}_{\mathrm{K}} x_{1}^{4}\left(y_{2}-V_{\mathrm{K}}\right)-\bar{g}_{\mathrm{Na}} \bar{y}_{1}^{3} x_{2}\left(y_{2}-V_{\mathrm{Na}}\right) \\
& \quad-\bar{g}_{\mathrm{L}}\left(y_{2}-V_{\mathrm{L}}\right)=0, \\
& \frac{4 \bar{g}_{\mathrm{K}} x_{1}^{3}\left(y_{2}-V_{\mathrm{K}}\right)\left(x_{1}-\bar{x}_{1}\right)}{\tau_{1}} \\
& \quad+\frac{\bar{g}_{\mathrm{Na}} \bar{y}_{1}^{3}\left(y_{2}-V_{\mathrm{Na}}\right)\left(x_{2}-\bar{x}_{2}\right)}{\tau_{2}}=0, \\
& \bar{g}_{\mathrm{K}} x_{1}^{4}+\bar{g}_{\mathrm{Na}} \bar{y}_{1}^{3} x_{2}+\bar{g}_{\mathrm{L}}+3 \bar{g}_{\mathrm{Na}} \bar{y}_{1}^{2} \bar{y}_{1}^{\prime} x_{2}\left(y_{2}-V_{\mathrm{Na}}\right)=0 .
\end{aligned}
$$

Moreover, (71a) and (71c) indicate that the pseudo singular point belongs to the slow manifold and to the fold curve. So, let us replace in (71b) the variables $x_{1}$ and $x_{2}$ by the variables $x_{1 f}$ and $x_{2 f}$ given by (68) and (69), respectively, which represent the parametric equations of fold curve

$$
\begin{aligned}
\frac{4 g_{\mathrm{K}} x_{1 f}^{3}\left(y_{2}-V_{\mathrm{K}}\right)\left(x_{1 f}-\bar{x}_{1}\right)}{\tau_{1}} & \\
+\frac{\bar{y}_{1}^{3}\left(y_{2}-V_{\mathrm{Na}}\right)\left(x_{2 f}-\bar{x}_{2}\right)}{\tau_{2}} & =0 .
\end{aligned}
$$

Thus, it appears that (72) depends on the variable $y_{2}$, on the functions gates dynamics timescales $\tau_{1}\left(y_{2}\right)$ and $\tau_{2}\left(y_{2}\right)$, and on the bifurcation parameter $\bar{I}$. According to Rubin and Wechselberger [26], the function $y_{2}(\bar{I})$, solution of (72), is independent of time multiplicative constants $k_{1}$ and $k_{2}$ that one could set in factor of $\tau_{1}\left(y_{2}\right)$ and $\tau_{2}\left(y_{2}\right)$.

So, following their works, let us plot the function $y_{2}(\bar{I})$ solution of (72) for various values of these time constants by posing successively in (72) $k_{1}=1,3,4.75$ and 7 and while fixing $k_{2}=1$. The result is presented in Figure 2 .

Let us notice that this plot (the function $y_{2}(\bar{I})$ solution of (71a), (71b), and (71c) has been plotted with Mathematica ${ }^{\circ}$

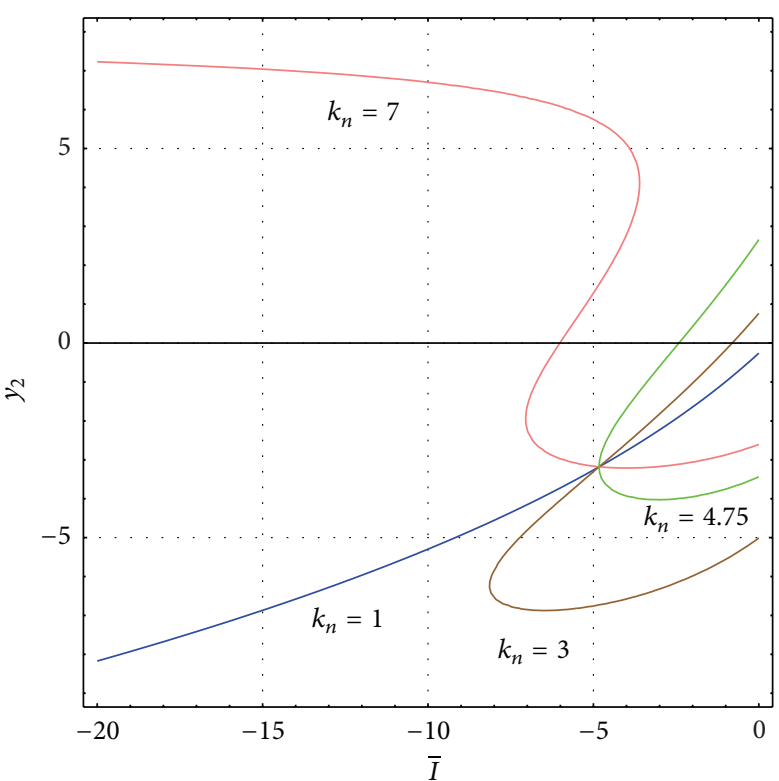

FIgURE 2: Function $y_{2}(\bar{I})$ for various values of parameter $k_{n}=$ $1,3,4.75,7$ exhibiting the the bifurcation parameter value $\bar{I}_{C} \approx$ -4.8 .

while using the Contour Plot Function used for representing implicit function since such function cannot be expressed explicitly) is exactly the same as those presented by Rubin and Wechselberger [26] (Fig. 8-9) for a nondimensionalized three-dimensional Hodgkin-Huxley singularly perturbed system which had been obtained after the following variable changes: $V \rightarrow-V$ and $\bar{I} \rightarrow-I$; then $V \rightarrow V+65$ and finally $V \rightarrow V / 100$.

We observe from Figure 2 that the bifurcation parameter value $\bar{I}_{C} \approx-4.8$ is exactly identical (in absolute value) to those obtained by Rubin and Wechselberger [26]. Numerical resolution (this resolution has been made while using the function FindRoot in Mathematica ${ }^{\odot}$ ) of (72) provides a better approximation of the bifurcation parameter value:

$$
\bar{I}_{C}=-4.82988 \mu \mathrm{A} \text {. }
$$

This value corresponds to a voltage $y_{2}=-3.18136 \mathrm{mV}$.

For $\bar{I} \approx-4.1$, the coordinate of the pseudo singular point can be computed numerically:

$$
\begin{aligned}
& \left(x_{1}, x_{2}, y_{1}, y_{2}\right) \\
& \quad=(0.362513,0.521793,0.0733782,-2.81908) .
\end{aligned}
$$

According to Proposition 3 we can state that the eigenpolynomial of the Jacobian matrix associated with the "normalized slow dynamics" of the Hodgkin-Huxley model (60a), (60b), (60c), and (60d) reads

$$
\lambda^{4}-\sigma_{1} \lambda^{3}+\sigma_{2} \lambda^{2}-\sigma_{3} \lambda+\sigma_{4}=0
$$

for which it is easy to prove that $\sigma_{4}=\sigma_{3}=0$. So, this eigenpolynomial reduces to

$$
\lambda^{2}\left(\lambda^{2}-\sigma_{1} \lambda+\sigma_{2}\right)=0 .
$$




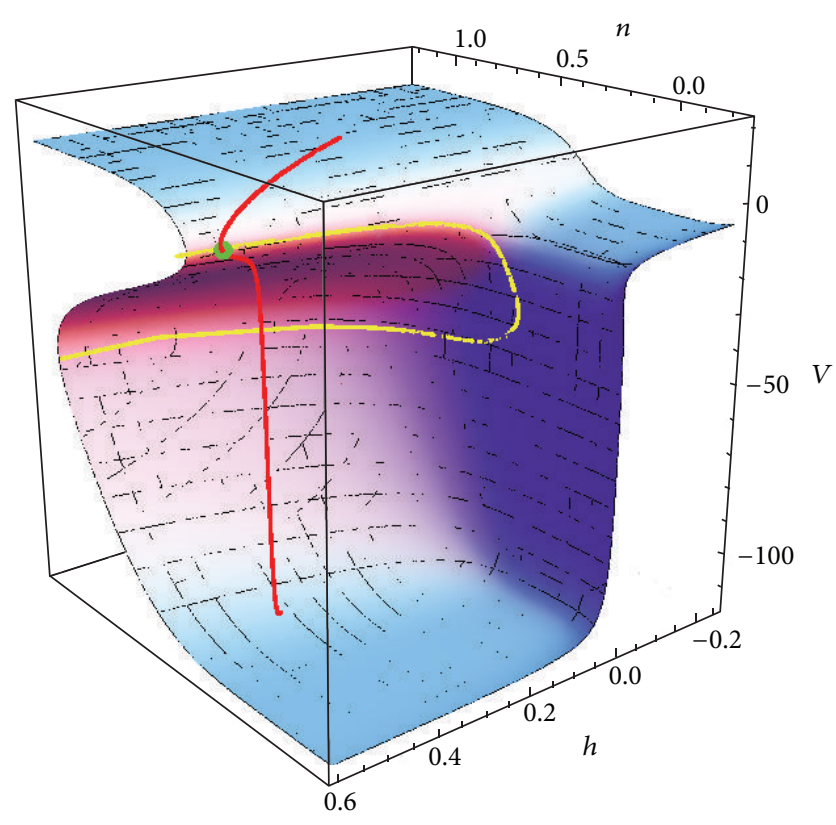

FIGURE 3: Phase portrait, canard solution, and slow manifold of the Hodgkin-Huxley system (60a), (60b), (60c), and (60d) in the ( $n, h$, $V)$ phase space.

According to (31) we find that

$$
\begin{aligned}
& p=\operatorname{Tr}(J)=144.933 \\
& q=\sigma_{2}=-362.924 .
\end{aligned}
$$

Thus, according to Proposition 3, the pseudo singular point is of saddle-type. Moreover, numerical computation of the eigenvalues of this Jacobian matrix evaluated at the pseudo singular point provides

$$
\left(\lambda_{1}, \lambda_{2}, \lambda_{3}, \lambda_{4}\right)=(-2.46224,147.396,0,0) .
$$

So, according to Proposition 3, this pseudo singular point is of saddle-type and canard solution may occur in the fourdimensional Hodgkin-Huxley singularly perturbed system (60a), (60b), (60c), and (60d) for the original set of parameter values.

In Figures 3, 4, and 5 canard solution of the fourdimensional Hodgkin-Huxley singularly perturbed system for the "canard value" of $\bar{I} \approx-4.1$ has been plotted in the $\left(x_{1}, x_{2}, y_{2}\right)$ phase space and then in the $\left(x_{1}, y_{2}\right)$ phase plane. The green point represents the pseudo singular point. The trajectory curve, that is, the canard solution, has been plotted in red while the fold curve is in yellow. We observe in Figure 3 that when the trajectory curve reaches the fold at the pseudo singular point it "jumps" suddenly to the other part of the slow manifold before being reinjected towards the pseudo singular point.

\section{Discussion}

In a previous paper entitled "Canards Existence in Memristor's Circuits" (see Ginoux and Llibre [12]) we have proposed

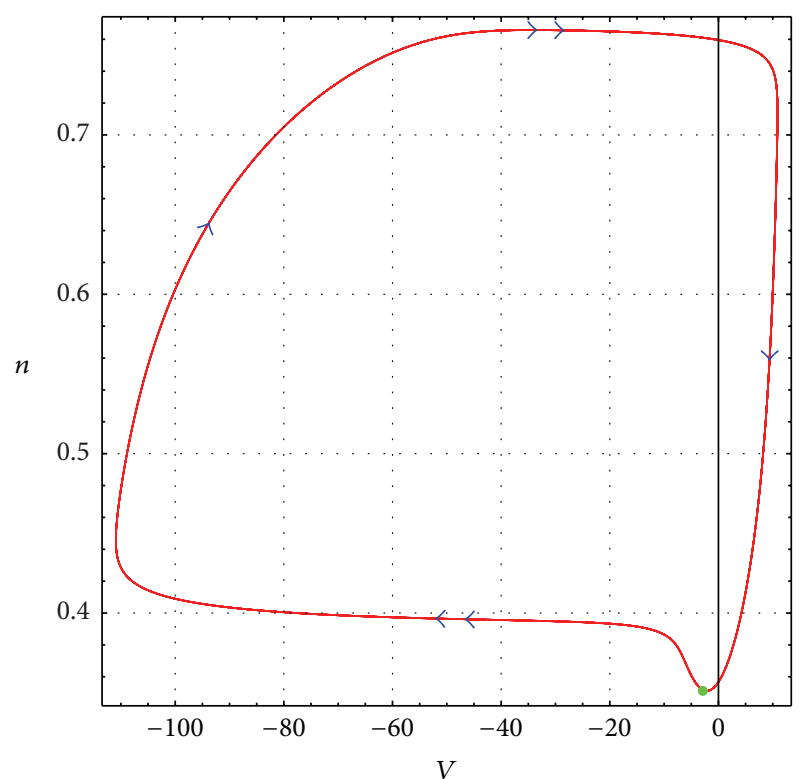

FIGURE 4: Phase portrait, canard solution, and slow manifold of the Hodgkin-Huxley system (60a), (60b), (60c), and (60d) in the $(V, n)$ phase plane.

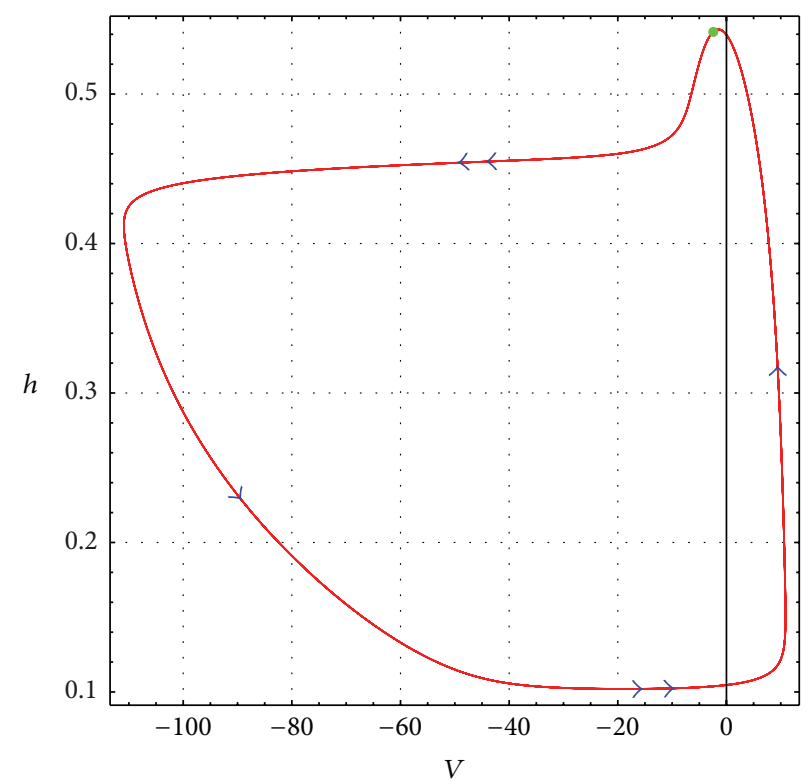

FIGURE 5: Phase portrait, canard solution, and slow manifold of the Hodgkin-Huxley system (60a), (60b), (60c), and (60d) in the $(V, h)$ phase plane.

a new method for proving the existence of "canard solutions" for three- and four-dimensional singularly perturbed systems with only one fast variable which improves the methods used until now. This method enabled stating a unique "generic" condition for the existence of "canard solutions" for such three- and four-dimensional singularly perturbed systems which is based on the stability of folded singularities of the normalized slow dynamics deduced from a well-known property of linear algebra. This unique condition which is 
completely identical to that provided by Benoît [2] and then by Szmolyan and Wechselberger [5] and finally by Wechselberger [11] was considered as "generic" since it was exactly the same for singularly perturbed systems of dimensions three and four with only one fast variable. In this work we have extended this new method to the case of four-dimensional singularly perturbed systems with two slow and two fast variables and we have stated that the condition for the existence of "canard solutions" in such systems is exactly identical to those proposed in our previous paper. This result confirms the genericity of the condition $\left(\sigma_{2}<0\right)$; we have highlighted and provide a simple and efficient tool for testing the occurrence of "canard solutions" in any three- or four-dimensional singularly perturbed systems with one or two fast variables. Applications of this method to the famous coupled FitzHugh-Nagumo equations and to the HodgkinHuxley model has enabled showing the existence of "canard solutions" in such systems. However, in this paper, only the case of pseudo singular points or folded singularities of saddletype has been analyzed. Of course, the case of of pseudo singular points or folded singularities of node-type and focustype could be also studied with the same method.

\section{Appendix}

Changes of coordinates leading to the normal forms of four-dimensional singularly perturbed systems with two fast variables are given in the following section.

\section{Normal Form of 4D Singularly Perturbed Systems with Two Fast Variables}

Let us consider the four-dimensional singularly perturbed dynamical system (13a), (13b), (13c), and (13d) with $k=2$ slow variables and $m=2$ fast and let us make the following change of variables:

$$
\begin{aligned}
& x_{1}=\alpha^{2} x, \\
& x_{2}=\alpha y, \\
& y_{1}=\alpha^{2} z, \\
& y_{2}=\alpha u,
\end{aligned}
$$

where $\alpha \ll 1$.

By taking into account extension of Benoît's generic hypothesis ((24), (25)) and while using Taylor series expansion, system (13a), (13b), (13c), and (13d) becomes

$$
\begin{aligned}
\dot{x} & =\frac{\partial f_{1}}{\partial y} y+\frac{\partial f_{1}}{\partial u} u, \\
\dot{y} & =f_{2}(x, y, z, u), \\
\left(\frac{\varepsilon}{\alpha}\right) \dot{z} & =\frac{\partial g_{1}}{\partial z} z+\frac{1}{2} \frac{\partial^{2} g_{1}}{\partial y^{2}} y^{2}+\frac{1}{2} \frac{\partial^{2} g_{1}}{\partial u^{2}} u^{2}+\frac{\partial^{2} g_{1}}{\partial y \partial u} y u, \\
\left(\frac{\varepsilon}{\alpha}\right) \dot{u} & =\frac{\partial g_{2}}{\partial x} x+\frac{1}{2} \frac{\partial^{2} g_{2}}{\partial y^{2}} y^{2}+\frac{1}{2} \frac{\partial^{2} g_{2}}{\partial u^{2}} u^{2}+\frac{\partial^{2} g_{2}}{\partial y \partial u} y u .
\end{aligned}
$$

Then, let us make the standard polynomial change of variables:

$$
\begin{aligned}
& X=A x+B y^{2}, \\
& Y=\frac{y}{f_{2}}, \\
& Z=C y+D z+E u, \\
& U=F y+G u .
\end{aligned}
$$

From (A.3) we deduce that

$$
\begin{aligned}
& x=\frac{X-B f_{2}^{2} Y^{2}}{A}, \\
& y=f_{2} Y, \\
& z=\frac{1}{D}\left[Z-C f_{2} Y-\frac{E}{G}\left(U-F f_{2} Y\right)\right], \\
& u=\frac{U-F f_{2} Y}{G} .
\end{aligned}
$$

The time derivative of system (A.3) gives

$$
\begin{aligned}
& \dot{X}=A \dot{x}+2 B y \dot{y}, \\
& \dot{Y}=\frac{\dot{y}}{f_{2}}, \\
& \dot{Z}=C \dot{y}+D \dot{z}+E \dot{u}, \\
& \dot{U}=F \dot{z}+G \dot{u} .
\end{aligned}
$$

Then, multiplying the third and fourth equation of (A.5) by $(\varepsilon / \alpha)$ while replacing in (A.5) $\dot{x}, \dot{y}, \dot{z}$, and $\dot{u}$ by the righthand side of system (A.2) leads to

$$
\begin{aligned}
\dot{X} & =A \dot{x}+2 B y \dot{y}, \\
\dot{Y} & =\frac{\dot{y}}{f_{2}}, \\
\left(\frac{\varepsilon}{\alpha}\right) \dot{Z} & =\left(\frac{\varepsilon}{\alpha}\right) C \dot{y}+\left(\frac{\varepsilon}{\alpha}\right) D \dot{z}+\left(\frac{\varepsilon}{\alpha}\right) E \dot{u}, \\
\left(\frac{\varepsilon}{\alpha}\right) \dot{U} & =\left(\frac{\varepsilon}{\alpha}\right) F \dot{y}+\left(\frac{\varepsilon}{\alpha}\right) G \dot{u} .
\end{aligned}
$$

Since $\varepsilon / \alpha \ll 1$, the first terms of the right-hand side of the third and fourth equation of (A.6) can be neglected. So we have

$$
\begin{aligned}
& \dot{X}=A\left(\frac{\partial f_{1}}{\partial y} y+\frac{\partial f_{1}}{\partial u} u\right)+2 B f_{2} y \\
& \dot{Y}=1 \\
& \left(\frac{\varepsilon}{\alpha}\right) \dot{Z}=D\left(\frac{\partial g_{1}}{\partial z} z+\frac{1}{2} \frac{\partial^{2} g_{1}}{\partial y^{2}} y^{2}+\frac{1}{2} \frac{\partial^{2} g_{1}}{\partial u^{2}} u^{2}\right. \\
& \left.\quad+\frac{\partial^{2} g_{1}}{\partial y \partial u} y u\right)+E\left(\frac{\partial g_{2}}{\partial x} x+\frac{1}{2} \frac{\partial^{2} g_{2}}{\partial y^{2}} y^{2}+\frac{1}{2} \frac{\partial^{2} g_{2}}{\partial u^{2}} u^{2}\right.
\end{aligned}
$$




$$
\begin{aligned}
& \left.+\frac{\partial^{2} g_{2}}{\partial y \partial u} y u\right) \\
& \left(\frac{\varepsilon}{\alpha}\right) \dot{U}=G\left(\frac{\partial g_{2}}{\partial x} x+\frac{1}{2} \frac{\partial^{2} g_{2}}{\partial y^{2}} y^{2}+\frac{1}{2} \frac{\partial^{2} g_{2}}{\partial u^{2}} u^{2}\right. \\
& \left.+\frac{\partial^{2} g_{2}}{\partial y \partial u} y u\right)
\end{aligned}
$$

Then, by replacing in (A.7) $x, y, z$, and $u$ by the right-hand side of (A.4) and by identifying with the following system in which we have posed $(\varepsilon / \alpha)=\epsilon$ :

$$
\begin{aligned}
\dot{X}= & \widetilde{a} Y+\widetilde{b} U+O\left(X, \epsilon, Y^{2}, Y U, U^{2}\right) \\
\dot{Y}= & 1+O(X, Y, U, \epsilon) \\
\epsilon \dot{Z}= & \tilde{c} Z+O\left(\epsilon X, \epsilon Y, \epsilon U, X^{2}, y^{2} U, U^{2}, Y U\right), \\
\epsilon \dot{U}= & -\left(X+U^{2}\right) \\
& +O\left(\epsilon X, \epsilon Y, \epsilon U, \epsilon^{2}, X^{2} U, U^{3}, X Y U\right),
\end{aligned}
$$

we find

$$
\begin{aligned}
\widetilde{a} & =A\left(\frac{\partial f_{1}}{\partial y}-\frac{F}{G} \frac{\partial f_{1}}{\partial u}\right) f_{2}+2 B f_{2}^{2}, \\
\widetilde{b} & =\frac{A}{G} \frac{\partial f_{1}}{\partial u} \\
\tilde{c} & =\frac{\partial g_{1}}{\partial z}
\end{aligned}
$$

where

$$
\begin{aligned}
& A=\frac{1}{2} \frac{\partial g_{2}}{\partial x} \frac{\partial^{2} g_{2}}{\partial u^{2}} \\
& B=\frac{1}{4}\left[\frac{\partial^{2} g_{2}}{\partial u^{2}} \frac{\partial^{2} g_{2}}{\partial y^{2}}-\left(\frac{\partial^{2} g_{2}}{\partial y \partial u}\right)^{2}\right], \\
& G=-\frac{1}{2} \frac{\partial^{2} g_{2}}{\partial u^{2}} .
\end{aligned}
$$

Finally, we deduce

$$
\begin{aligned}
\tilde{a} & =\frac{1}{2}\left[f_{2}^{2}\left(\frac{\partial^{2} g_{2}}{\partial x_{2}^{2}} \frac{\partial^{2} g_{2}}{\partial y_{2}^{2}}-\left(\frac{\partial^{2} g_{2}}{\partial x_{2} \partial y_{2}}\right)^{2}\right)\right. \\
& \left.+f_{2} \frac{\partial g_{2}}{\partial x_{1}}\left(\frac{\partial^{2} g_{2}}{\partial y_{2}^{2}} \frac{\partial f_{1}}{\partial x_{2}}-\frac{\partial^{2} g_{2}}{\partial x_{2} \partial y_{2}} \frac{\partial f_{1}}{\partial y_{2}}\right)\right], \\
\widetilde{b} & =-\frac{\partial g_{2}}{\partial x_{1}} \frac{\partial f_{1}}{\partial y_{2}} \\
\tilde{c} & =\frac{\partial g_{1}}{\partial y_{1}} .
\end{aligned}
$$

This is the result we established in Section 2.7.

\section{Conflict of Interests}

The authors declare that there is no conflict of interests regarding the publication of this paper.

\section{Acknowledgments}

One of the authors (Jaume Llibre) is partially supported by a MINECO Grant MTM2013-40998-P, an AGAUR Grant no. 2014SGR-568, and Grants FP7-PEOPLE-2012-IRSES 318999 and 316338.

\section{References}

[1] E. Benoît and C. Lobry, "Les canards de $\mathbb{R}^{3}$," Comptes Rendus de l'Académie des Sciences Series I, vol. 294, pp. 483-488, 1982.

[2] E. Benoît, "Systémes lents-rapides dans $\mathbb{R}^{3}$ et leurs canards," Astérisque, Société Mathématique de France, vol. 109-110, no. 2, pp. 159-191, 1983.

[3] E. Benoît, Canards de $\mathbb{R}^{3}$ [Thése d'État $(P h D)$ ], Université de Nice, Nice, France, 1984.

[4] J. Argémi, "Approche qualitative d'un probléme de perturbations singuliéres dans $\mathbb{R}^{4}$," in Equadiff 1978, R. Conti, G. Sestini, and G. Villari, Eds., pp. 330-340, 1978.

[5] P. Szmolyan and M. Wechselberger, "Canards in $\mathbb{R}^{3}$," Journal of Differential Equations, vol. 177, no. 2, pp. 419-453, 2001.

[6] N. Fenichel, "Persistence and smoothness of invariant manifolds for flows," Indiana University Mathematics Journal, vol. 21, pp. 193-226, 1971.

[7] N. Fenichel, "Geometric singular perturbation theory for ordinary differential equations," Journal of Differential Equations, vol. 31, no. 1, pp. 53-98, 1979.

[8] R. E. O'Malley, Introduction to Singular Perturbations, Academic Press, New York, NY, USA, 1974.

[9] C. K. R. T. Jones, "Geometric singular perturbation theory," in Dynamical Systems, vol. 1609 of Lecture Notes in Mathematics, pp. 44-118, Springer, 1994.

[10] T. Kaper, "An introduction to geometric methods and dynamical systems theory for singular perturbation problems," in Analyzing Multiscale Phenomena Using Singular Perturbation Methods, pp. 85-131, American Mathematical Society, Providence, RI, USA, 1998.

[11] M. Wechselberger, "À propos de canards," Transactions of the American Mathematical Society, vol. 364, pp. 3289-3309, 2012.

[12] J. M. Ginoux and J. Llibre, "Canards existence in memristor's circuits," Qualitative Theory of Dynamical Systems, pp. 1-49, 2015.

[13] A. N. Tikhonov, "On the dependence of solutions of differential equations on a small parameter," Matematicheskii Sbornik, vol. 31, pp. 575-586, 1948.

[14] E. Benoît, "Perturbation singuliére en dimension trois: canards en un point pseudo singulier noeud," Bulletin de la Société Mathématique de France, vol. 129, no. 1, pp. 91-113, 2001.

[15] M. Wechselberger, "Existence and bifurcation of canards in $\mathbb{R}^{3}$ in the case of a folded node," SIAM Journal on Applied Dynamical Systems, vol. 4, no. 1, pp. 101-139, 2005.

[16] F. Takens, "Constrained equations; a study of implicit differential equations and their discontinuous solutions," in Structural Stability, the Theory of Catastrophes, and Applications in the Sciences, vol. 525 of Lecture Notes in Mathematics, pp. 143-234, Springer, Berlin, Germany, 1976. 
[17] J. Guckenheimer and R. Haiduc, "Canards at folded nodes," Moscow Mathematical Journal, vol. 5, no. 1, pp. 91-103, 2005.

[18] K. Tchizawa and S. A. Campbell, "On winding duck solutions in $\mathbb{R}^{4}$," Proceedings of Neural, Parallel, and Scientific Computations, vol. 2, pp. 315-318, 2002.

[19] K. Tchizawa, "On duck solutions in $\mathbb{R}^{4}$," Kyoto UniversityResearch Institute for Mathematical Sciences: Kokyuroku, vol. 1254, pp. 160-162, 2002.

[20] K. Tchizawa, "A direct method for finding ducks in $\mathbb{R}^{4}$," RIMS Kokyuroku, vol. 1372, pp. 97-103, 2004.

[21] K. Tchizawa, "Generic conditions for duck solutions in $\mathbb{R}^{4}$," RIMS Kokyuroku, vol. 1547, pp. 107-113, 2007.

[22] K. Tchizawa, "On the transversality conditions for 4-dim duck solutions," RIMS Kokyuroku, vol. 1582, pp. 144-150, 2008.

[23] K. Tchizawa, "On a local model for finding 4-dimensional duck solutions," in Selected Topics in Mathematical Methods and Computational Techniques in Electrical Engineering, pp. 177-183, 2010.

[24] K. Tchizawa, "On relative stability in 4-dimensional duck solution," Journal of Mathematics and System Science, no. 2, pp. 558-563, 2012.

[25] K. Tchizawa, "On the two methods for finding 4-dimensional duck solutions," Applied Mathematics, vol. 5, no. 1, pp. 16-24, 2014.

[26] J. Rubin and M. Wechselberger, "Giant squid-hidden canard: the $3 \mathrm{D}$ geometry of the Hodgkin-Huxley model," Biological Cybernetics, vol. 97, no. 1, pp. 5-32, 2007.

[27] L. O. Chua, V. I. Sbitnev, and H. Kim, "Hodgkin-Huxley axon is made of memristors," International Journal of Bifurcation and Chaos, vol. 22, no. 3, Article ID 1230011, 2012.

[28] L. O. Chua, V. I. Sbitnev, and H. Kim, "Neurons are poised near the edge of chaos," International Journal of Bifurcation and Chaos, vol. 22, no. 4, Article ID 1250098, 2012.

[29] R. FitzHugh, "Impulses and physiological states in theoretical models of nerve membrane," Biophysical Journal, vol. 1, no. 6, pp. 445-466, 1961.

[30] J. S. Nagumo, S. Arimoto, and S. Oshizawa, "An active pulse transmission line simulating nerve axon," Proceedings of the Institute of Radio Engineers, vol. 50, no. 10, pp. 2061-2070, 1962.

[31] A. L. Hodgkin and A. F. Huxley, "A quantitative description of membrane current and its application to conduction and excitation in nerve," The Journal of physiology, vol. 117, no. 4, pp. 500-544, 1952.

[32] R. Suckley and V. N. Biktashev, "The asymptotic structure of the Hodgkin-Huxley equations," International Journal of Bifurcation and Chaos, vol. 13, no. 12, pp. 3805-3825, 2003.

[33] R. Suckley, The asymptotic structure of excitable systems [Ph.D. thesis], University of Liverpool, 2004.

[34] F. Awiszus, J. Dehnhardt, and T. Funke, "The singularly perturbed Hodgkin-Huxley equations as a tool for the analysis of repetitive nerve activity," Journal of Mathematical Biology, vol. 28, no. 2, pp. 177-195, 1990. 


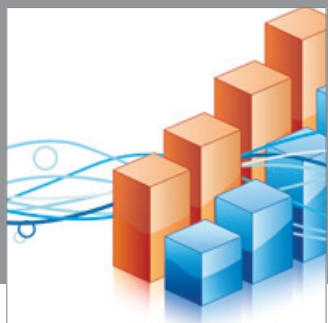

Advances in

Operations Research

mansans

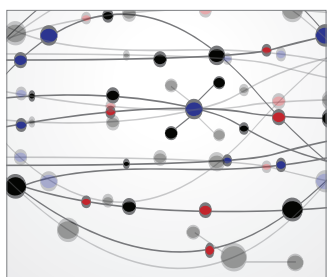

The Scientific World Journal
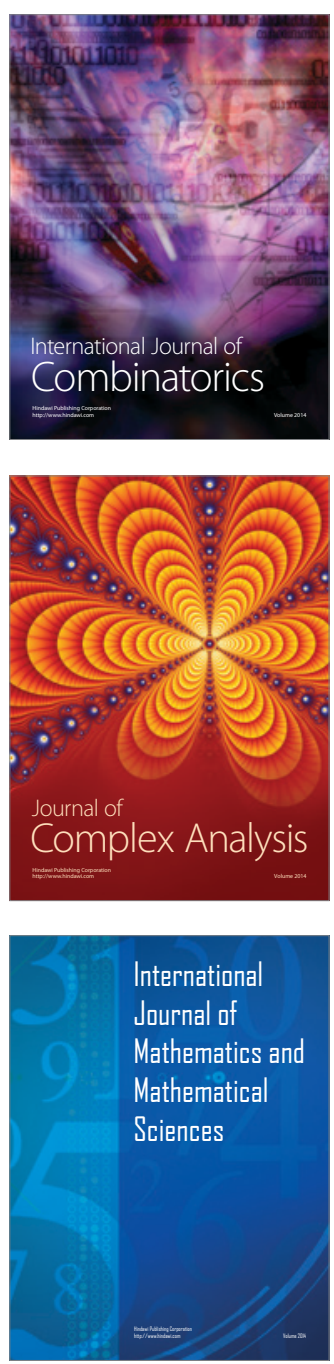
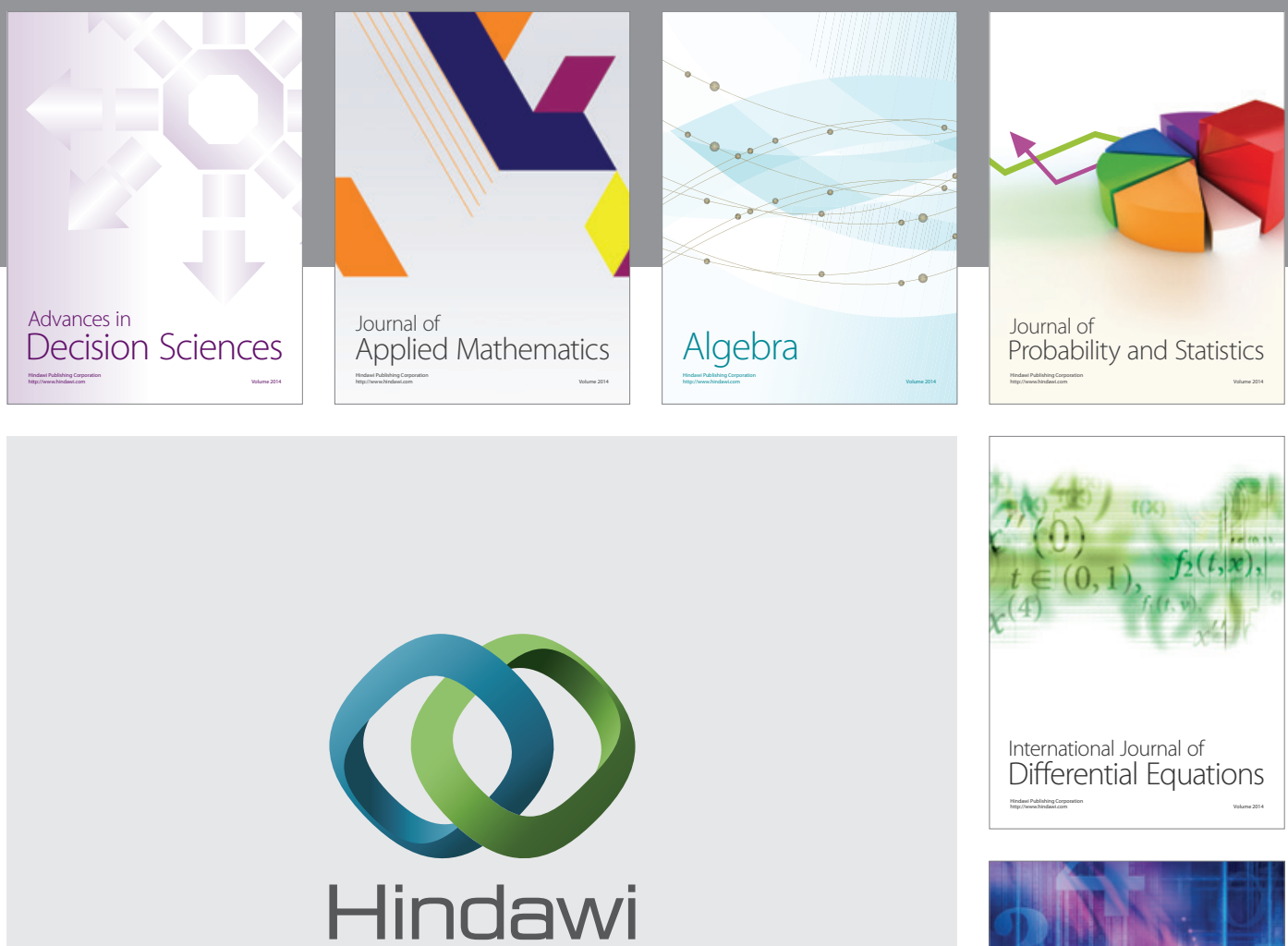

Submit your manuscripts at http://www.hindawi.com
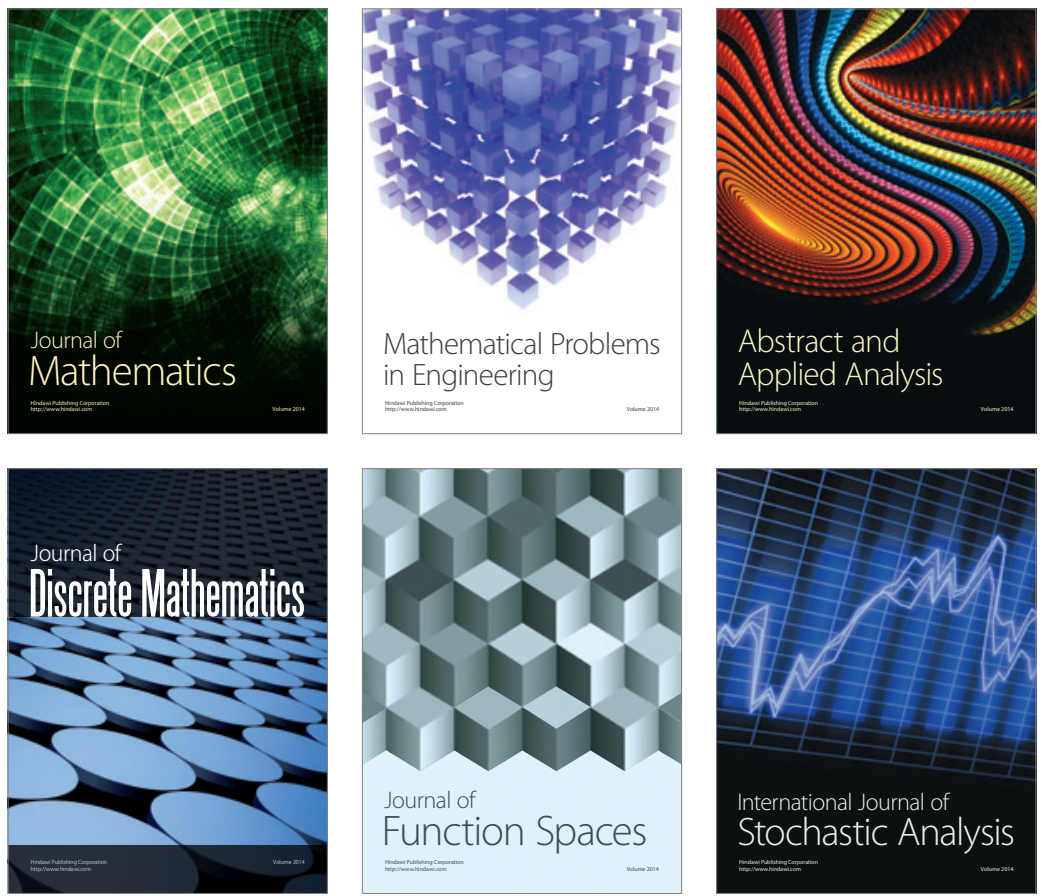

Journal of

Function Spaces

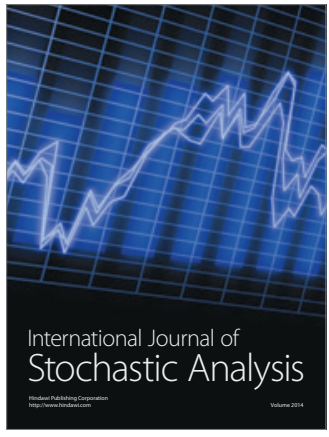

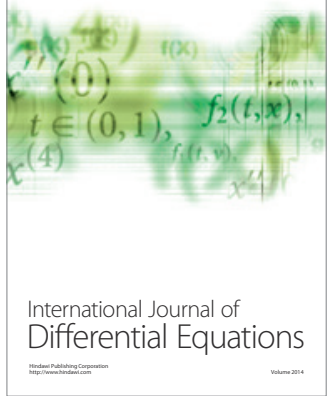
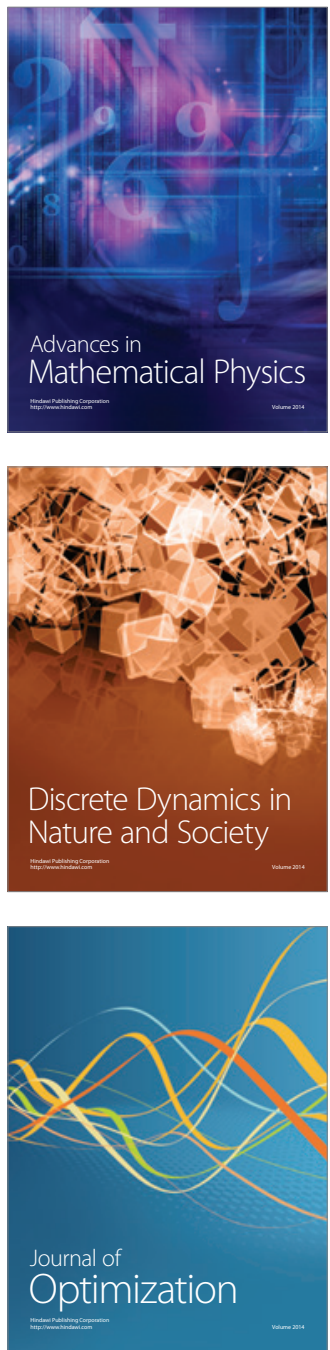